\title{
AGR-1 Thermocouple Data Analysis
}

Binh T. Pham Jeffrey J. Einerson

May 2012

The INL is a

U.S. Department of Energy National Laboratory

operated by

Battelle Energy Alliance

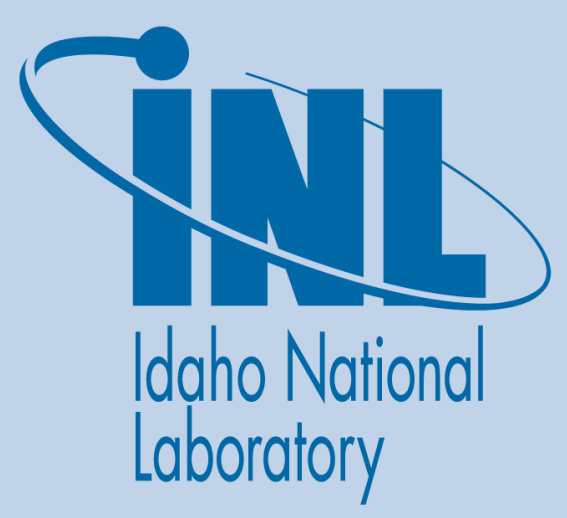

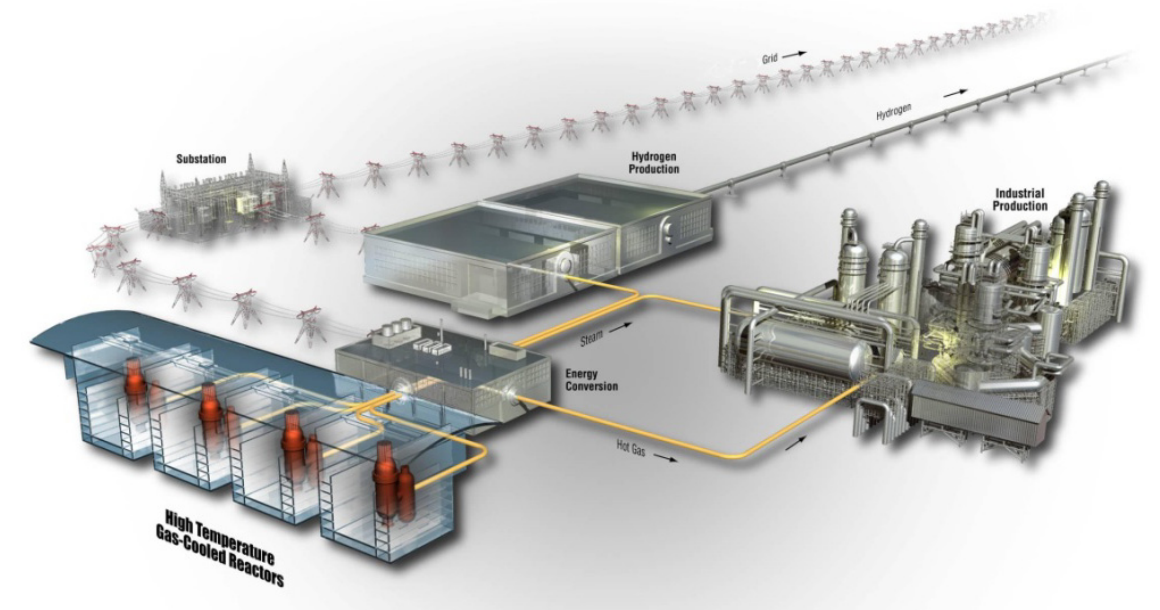




\section{DISCLAIMER}

This information was prepared as an account of work sponsored by an agency of the U.S. Government. Neither the U.S. Government nor any agency thereof, nor any of their employees, makes any warranty, expressed or implied, or assumes any legal liability or responsibility for the accuracy, completeness, or usefulness, of any information, apparatus, product, or process disclosed, or represents that its use would not infringe privately owned rights. References herein to any specific commercial product,

process, or service by trade name, trade mark, manufacturer, or otherwise, does not necessarily constitute or imply its endorsement, recommendation, or favoring by the U.S. Government or any agency thereof. The views and opinions of authors expressed herein do not necessarily state or reflect those of the U.S. Government or any agency thereof. 


\title{
AGR-1 Thermocouple Data Analysis
}

\author{
Binh T. Pham \\ Jeffrey J. Einerson
}

May 2012

\begin{abstract}
Idaho National Laboratory
Next Generation Nuclear Plant Project

Idaho Falls, Idaho 83415
\end{abstract}

http://www.inl.gov

\section{Prepared for the}

U.S. Department of Energy

Office of Nuclear Energy

Under DOE Idaho Operations Office

Contract DE-AC07-05ID14517 


\title{
Next Generation Nuclear Plant Project
}

\section{AGR-1 Thermocouple Data Analysis}

\author{
INL/EXT-12-24761
}

May 2012

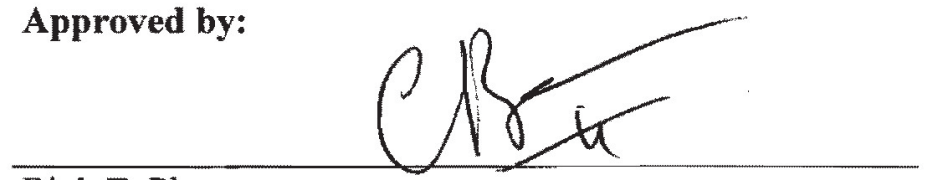

Binh T. Pham

Author

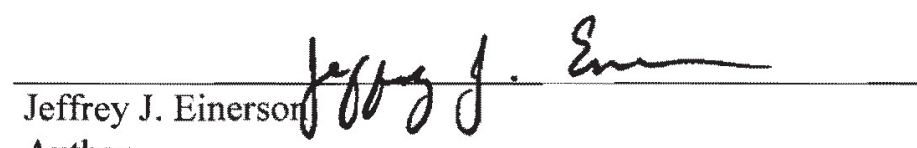

Author

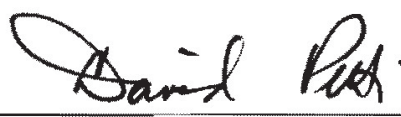

David A. Petti

VHTR TDQPirector

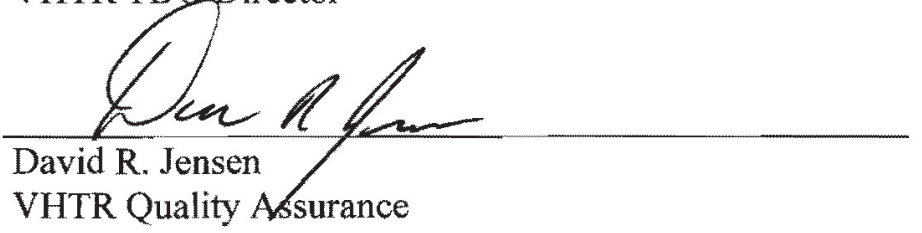

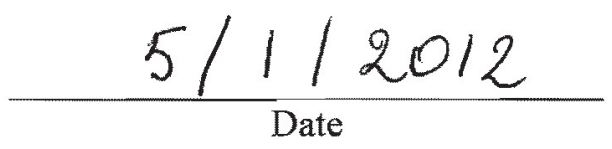

$5-1-2012$ Date

$\frac{5-1-2012}{\text { Date }}$

$5 / 1 / 12$ 


\begin{abstract}
This report documents an analysis of measured and simulated data obtained in the Advanced Gas Reactor (AGR) fuel irradiation test program conducted in the Advanced Test Reactor at Idaho National Laboratory to support the Very High Temperature Reactor (VHTR) Research and Development program. The work follows a previous study done by Pham and Einerson (2010), in which statistical analysis methods were applied for AGR-1 thermocouple data qualification. The present work exercises the idea that, while recognizing uncertainties inherent in physics and thermal simulations of the AGR-1 test, results of the numerical simulations can be used in combination with the statistical analysis methods to further improve qualification of measured data. The combined analysis of measured and simulation data can also generate insights about simulation model uncertainty that can be useful for model improvement.
\end{abstract}

This report also describes an experimental control procedure to maintain fuel target temperature in the future AGR tests using regression relationships that include simulation results. 


\section{SUMMARY}

This report documents an analysis of measured and simulated data obtained in the Advanced Gas Reactor (AGR) fuel irradiation test program conducted in the Advanced Test Reactor at Idaho National Laboratory to support the Very High Temperature Reactor (VHTR) Research and Development program. The work follows a previous study done by Pham and Einerson (2010), in which statistical analysis methods were applied for AGR-1 thermocouple data qualification. The present work exercises the idea that, while recognizing uncertainties inherent in physics and thermal simulations of the AGR-1 test, results of the numerical simulations can be used in combination with the statistical analysis methods to further improve qualification of measured data. The combined analysis of measured and simulation data can also generate insights about simulation model uncertainty that can be useful for model improvement.

This report also describes an experimental control procedure to maintain fuel target temperature in the future AGR tests using regression relationships that include simulation results.

The report is organized into four sections:

- Section 1 introduces the AGR Fuel Development and Qualification Program's AGR-1 test configuration and test procedure, overviews AGR-1 measured data, and overviews physics and thermal simulation, including modeling assumptions and uncertainties. A brief summary of statistical analysis methods developed by Pham and Einerson (2010) for AGR-1 measured data qualification within the NGNP Data Management and Analysis System is also included for completeness.

- Sections 2 and 3 describe and discuss cases, in which the combined use of experimental and simulation data is realized. A set of issues associated with measurement and modeling uncertainties resulting from the combined analysis are identified. This includes demonstrating that such a combined analysis led to important insights for reducing uncertainty in presentation of AGR-1 measured data (Section 2) and interpretation of simulation results (Section 3).

- The statistics-based simulation-aided experimental control procedure described for the future AGR tests is developed and demonstrated in Section 4 . The procedure for controlling the target fuel temperature (capsule peak or average) is based on regression functions of thermocouple readings and other relevant parameters, accounting for possible changes in both physical and thermal conditions and in instrument performance. 


\section{CONTENTS}

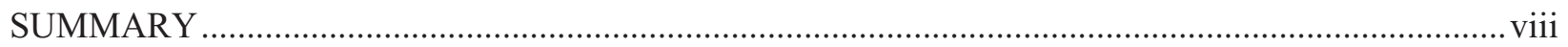

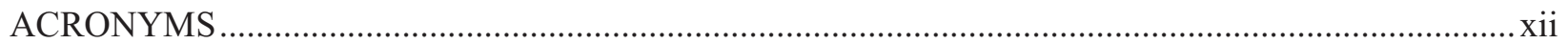

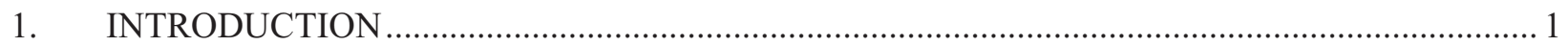

$1.1 \quad$ NGNP Fuel Development and Qualification Experimental Program …................................ 1

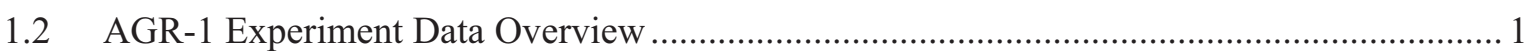

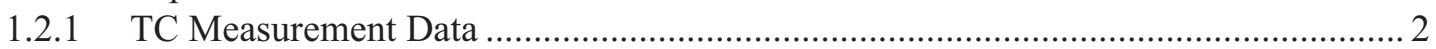

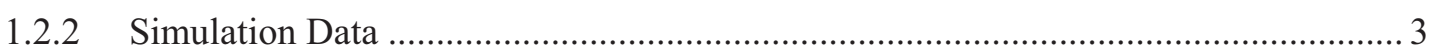

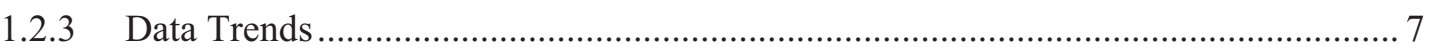

1.3 Statistical Analysis Methods for AGR Data Qualification …............................................. 9

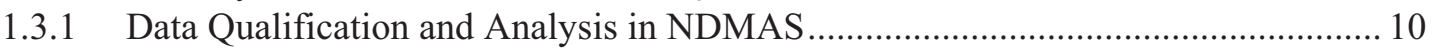

1.3.2 Statistical Methods for Data Accuracy Monitoring .............................................. 11

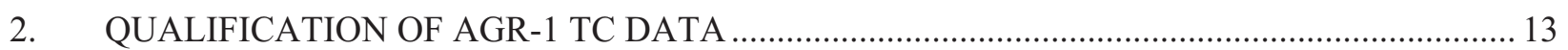

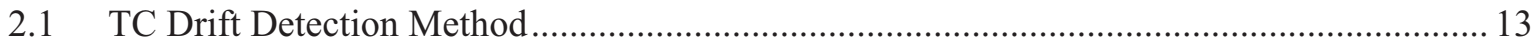

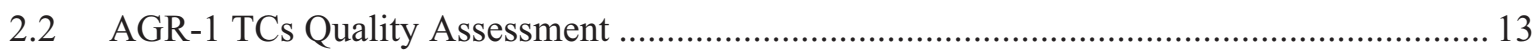

2.2.1 Capsule 6 (TC3, 4, and 5 are stable, but biased down by more than $100^{\circ} \mathrm{C}$;

$\mathrm{TC} 1$ and 2 are drifted down during the last two cycles) ........................................ 14

2.2.2 Capsule 5 (TC1 drifted upward by $200^{\circ} \mathrm{C}$ and TC3 is stable with wide variation)

2.2.3 Capsule 4 (TC1 and TC3 were stable and TC2 drifted down by more than $\left.100^{\circ} \mathrm{C}\right)$.

2.2.4 Capsule 3 (TC1 is biased up before failure date, TC2 drifted and failed during the second cycle, and TC3 is more or less stable in relation to

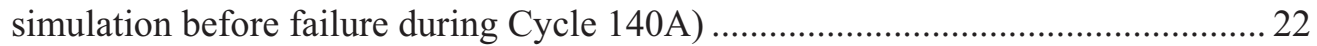

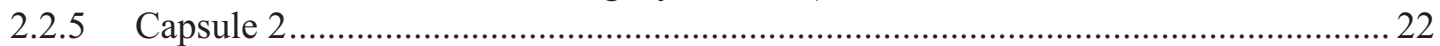

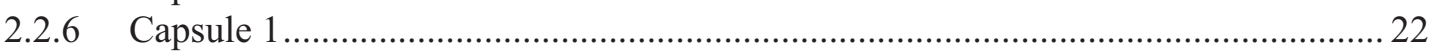

2.3 AGR-1 TC Characteristic and Performance Summary ...................................................... 25

2.3.1 Main Findings of AGR-1 TC Performance from Data Analysis .............................. 26

3. AGR-1 PHYSICS AND THERMAL SIMULATION DATA ANALYSIS ….............................2

3.1 Underestimation of Fission Power at the End of an ATR Cycle .........................................29

3.1.1 Upward TC Residual Trends for TC3 in Four Middle Capsules During the First Cycle ...................................................................................................... 29

3.1.2 TC Residual Upward Trend within Each ATR Cycle for TCs in Capsule 6.............29

3.1.3 JMOCUP Depletion Model May Underestimate Capsule Power at the End

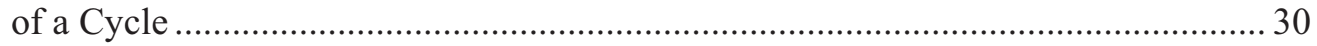

3.2 Overestimation of TC Temperature with Increasing Neon Fraction................................... 31

3.2.1 Evidence Based on Zoom-in of Irradiation Parameters Plots ................................. 31

3.2.2 Evidence Based on Regression Analysis .............................................................. 33

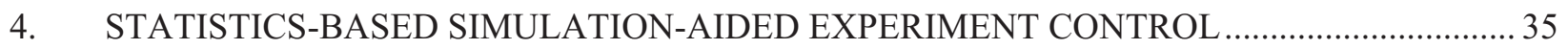

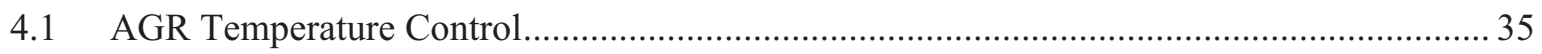

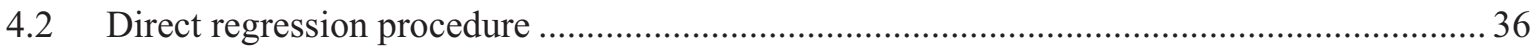




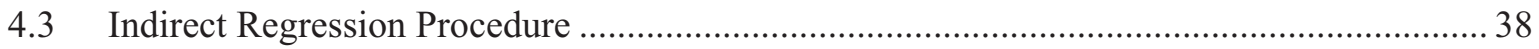

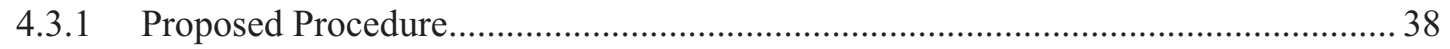

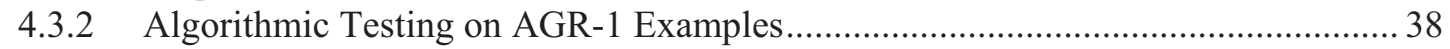

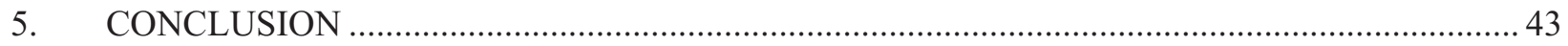

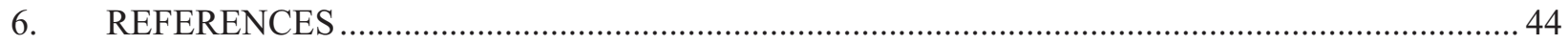

\section{FIGURES}

Figure 1. TC positions and types and their failure status for AGR-1 experiment..................................... 3

Figure 2. ATR operating parameters as function of AGR-1 EFPDs and ATR cycles............................... 5

Figure 3. Temperature $\left({ }^{\circ} \mathrm{C}\right)$ contour plot of cutaway view of three fuel stacks during cycle $141 \mathrm{~A} \ldots \ldots \ldots \ldots . .7$

Figure 4. Graphical summary of AGR-1 irradiation data for Capsule 6 .................................................... 9

Figure 5. NDMAS data qualification and analysis to support AGR tests................................................. 11

Figure 6. Thermocouple measured data accuracy monitoring in AGR experiment................................. 12

Figure 7. Histograms of temperature residuals of TC3, TC4, and TC5 of Capsule 6.............................. 14

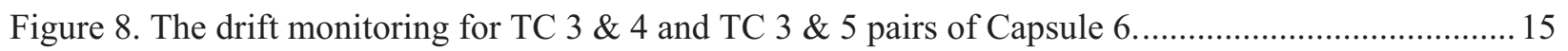

Figure 9. The drift monitoring for TC $1 \& 3$ and TC $2 \& 3$ pairs of Capsule 6..................................... 17

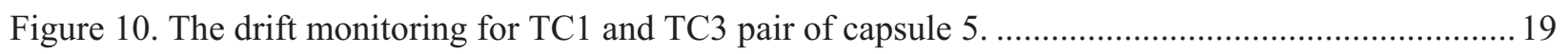

Figure 11. Histograms of simulated minus measured for TC1 and TC3 in capsule 5............................ 19

Figure 12. The drift monitoring for TC2 \& 1 and TC1 \& 3 pairs of Capsule 4 ........................................2

Figure 13. The drift monitoring for TC1 \& 2 and TC1 \& 3 pairs of Capsule 3........................................23

Figure 14. The drift monitoring for TC2 \& 3 pair of capsule 2 and TC2 of Capsule 1 ...........................2 24

Figure 15. TC characteristic and performance summary for AGR-1 experiment....................................2 25

Figure 16. Difference between measured and predicted TC temperatures. ...........................................28

Figure 17. TC1 and TC3 in Capsule 6 and relevant parameters data from 150 to 200 EFPDs................. 30

Figure 18. TC1 and TC3 in Capsule 5 and relevant parameters data from 240 to 300 EFPDs................. 32

Figure 19. TC1 and TC3 in Capsule 4 and relevant parameters data from 265 to 295 EFPDs..................33

Figure 20. Prediction profilers of Capsule 5 measured and simulated TC3 values and fuel

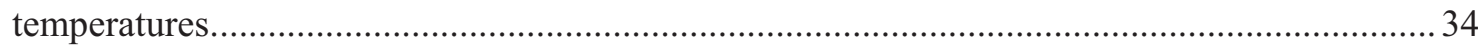

Figure 21. Capsule 4 measured and simulated TC1 values and fuel temperatures for all ATR

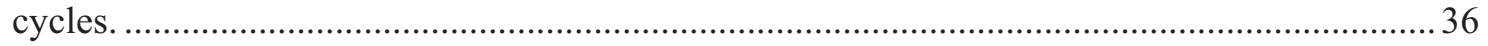

Figure 22. Capsule 6 peak fuel temperatures: regression (blue dots) and simulated (red dots)................. 37

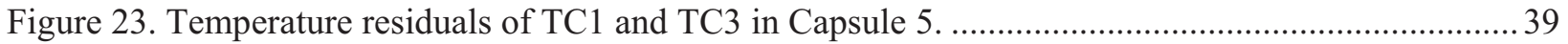

Figure 24. Prediction profilers for TC1 and simulated fuel temperature in Capsule 5 ........................... 40

Figure 25. Prediction profilers for TC3 and simulated fuel temperature in Capsule 5 ........................... 42 


\section{ACRONYMS}

AGR Advanced Gas Reactor

ATR Advanced Test Reactor

C center

CA correlation analysis

$\mathrm{CC} \quad$ control charting

ECAR Engineering Calculations and Analysis Reports

EFPD effective full-power day

INL Idaho National Laboratory

MCNP Monte Carlo N-Particle

NE northeast

NGNP Next Generation Nuclear Plant

NDMAS NGNP Data Management and Analysis System

OSCC outer shim control cylinders

PIE post-irradiation examination

RA regression analysis

$\mathrm{R} / \mathrm{B} \quad$ release-to-birthrate ratio

SAS Statistical Analysis System

SE southeast

TC thermocouple

VHTR Very High Temperature Reactor 


\section{AGR-1 Thermocouple Data Analysis}

\section{INTRODUCTION}

\subsection{NGNP Fuel Development and Qualification Experimental Program}

A series of Advanced Gas Reactor (AGR) irradiation experiments are being conducted within the VHTR R\&D Program for Next Generation Nuclear Plant (NGNP) fuel performance. These tests provide the needed fuel performance data to support fuel process development, qualify a fuel design and fabrication process for normal operation and accident conditions, and support development and validation of fuel performance and fission product transport models and codes (Petti, Hobbins, \& Kendal 2010; Simonds 2010). The fuel fabrication, irradiation, fission product monitoring, and post-irradiation examination (PIE) data collected from the AGR test campaign were imported into the NGNP Data Management and Analysis System (NDMAS) where it will be qualified and statistically analyzed (INL 2010). It is envisioned that both the experimental and computational data generated by the NGNP Project will be processed by state-of-the-art tools to ensure that the high-quality (and characteristically expensive) data remain accessible and usable by future generations of scientists and engineers. It is anticipated that the experimental data obtained in these tests will remain valuable, even several decades from now, for the assessment of models and codes developed to predict nuclear fuel performance that support fuel and plant licensing. As more advanced models and high-fidelity simulation methods become available in the future, their utility is expected to be even more sensitive to the knowledge about the quality of data used for model validation and uncertainty quantification. It is thus important that potentially misleading and deteriorated data from partially failed (drifted, conjunct) thermocouples (TCs) be accordingly identified.

Experimental data from NGNP fuel performance experiments are highly heterogeneous in nature. The AGR experimental data include local (measurement) and transient (measurement, simulation) data, integral (fission product release monitoring data from the fission product monitoring system) and accumulative (PIE) data. The different data types are, however, highly correlated as they reflect the same underlying physical process. This observation has led the authors to suggest using the strong correlation between experimental and simulation data to enable uncertainty reduction for both data streams: the simulation data can aid the accuracy testing in measurement data qualification and the experimental data is used for simulation code validation (e.g., the control gas mixture thermal conductivity model). Beyond the TC measurements, the accumulative data (e.g., from PIE) would help assess the accuracy of assumptions made in the ABAQUS (version 6.8-2) thermal model of the AGR capsules (e.g., the amount of graphite holder and fuel compact shrinkage under neutron irradiation, or the irradiation- and heatinduced component deformations leading to uneven regulation-gas gap).

It is also critical that the test conditions in AGR experiments be effectively maintained during the test and accurately characterized to ensure the experiments meet their objectives. The combination of both experimental and simulation data creates an opportunity to control the experiments, i.e., sustain the desired simulated quantities (e.g., calculated fuel temperature) by using their established relationship with measurements (e.g., controlled TC temperature) and other simulated parameters (e.g., fuel fission power density and graphite heat rate).

\subsection{AGR-1 Experiment Data Overview}

The AGR-1 test was conducted in the Advanced Test Reactor (ATR) at INL to obtain data on fuel behavior under normal and accident conditions of a new generation of very high-temperature gas-cooled 
reactors (VHTR) (Petti, Hobbins, \& Kendall 2010). The AGR test trains employed fuel compacts placed in a graphite cylinder shrouded by a steel capsule. Six capsules were used in the AGR-1 test numbered consecutively from the top, Capsule 6, to the bottom, Capsule 1 . The tests were instrumented with TCs embedded in graphite blocks. The target quantity (fuel compact temperature) was independently regulated by the helium-neon gas mixture that fills the gap volume for each capsule. The fission product releases of many radionuclides, which are important criteria for test fuel performance assessment, were separately monitored for each capsule. The AGR-1 test, which was inserted in the B10 position located on the east side of the ATR core in December 2006, was successfully completed in November 2009, resulting in fuel irradiation for a period of nearly 3 years or 620 effective full-power days (EFPDs). The AGR-1 data, including the TC readings collected over the course of irradiation (2007-2009), were scrutinized and stored in the NGNP Data Management and Analysis System. The primary objective of the AGR-1 test was the shakedown of the test train (Maki 2009). Therefore lessons learned from analysis of this test's extensive data are important for modification of subsequent test trains.

While no direct measurements of fuel temperature are available, the graphite temperature from TCs in AGR tests could be and were used to evaluate the target quantities, such as fuel temperature, as well as to validate computer codes. Besides TC temperatures, the helium-neon gas composition (or neon fraction of gas mixture) was also selected for this study. Qualification of these measurement data was based on the answer to the question: are the data reflecting what is expected or are anomalies or trends present that require investigation (Abbott et al. 2010). Simulation data were also needed because of the lack of direct measurement. Therefore, the NDMAS collected, organized (links data to appropriate components such as fuel compacts, capsules, etc.), and stored output from physics-based simulation codes, which calculated the ATR neutronics (and subsequently, power of capsule components) and thermal conditions (temperatures) of the AGR experiments. These simulated data were qualified by the modelers through Engineering Calculation and Analysis Reports (ECARs). The fission product release-to-birthrate ratios (R/Bs) for several isotopes (e.g., $\mathrm{Kr}-85 \mathrm{M}, \mathrm{Kr}-88$, and $\mathrm{Xe}-135$ ) were also included in this analysis because of their close relationship with temperature. The fission product $\mathrm{R} / \mathrm{B}$ is the ratio between measured radionuclide release rates (in atoms/sec) by the fission product monitoring system for each capsule and calculated radionuclide birthrates. These data were captured every 8 hours for use in confirming temperature changes.

\subsubsection{TC Measurement Data}

There were 17 TCs in six capsules where temperature in the graphite holder was recorded every 10 minutes for the initial phase of the experiment, then every 5 minutes for the remainder of the test. The number of TCs in capsules and the diameter of their wires were limited by space in the test train throughtubes. These TCs were selected based on the greatest survival probability and least amount of drift in accelerated furnace tests; TC failures were still expected because of high temperature and high neutron fluence exposure during extended irradiation far beyond vendor specification. Unfortunately, there was no calibration for the TCs after insertion in the AGR test train, even during PIE examination, so their reading accuracy could be assessed only by comparison with the readings from other TCs in similar positions or with simulated values. 
TC positions (on the capsule polar plane and insertion depth in graphite sample holder) and TC types and their failure status as recorded in the NDMAS data qualification report for AGR-1 experiment (Abbott et al. 2010) are shown in Figure 1. The blue colored TCs are INL fabricated molybdenumniobium type and the yellow colored TCs are commercial specialty Type-N TCs. The thick black rings surrounding TC3, TC4, and TC5 in Capsule 6 represent the thicker TC of 3/32-in.-diameter wire, while the rest of TCs are the thinner 1/16-in.-diameter wire. The red rings are for the TCs damaged at fabrication that had no qualified data, and the purple rings are for the TCs that failed during the course of the experiment that typically had qualified data from the beginning of the AGR-1 irradiation until the failure date determined by NGNP experts. By the end of the AGR-1 experiment, all TCs in Capsules 1, 2, and 3 and TC3 in Capsule 4 had failed, either at fabrication (TC1 in Capsules 1 and 2) or based on an assessment of their signals sometime during the irradiation period.

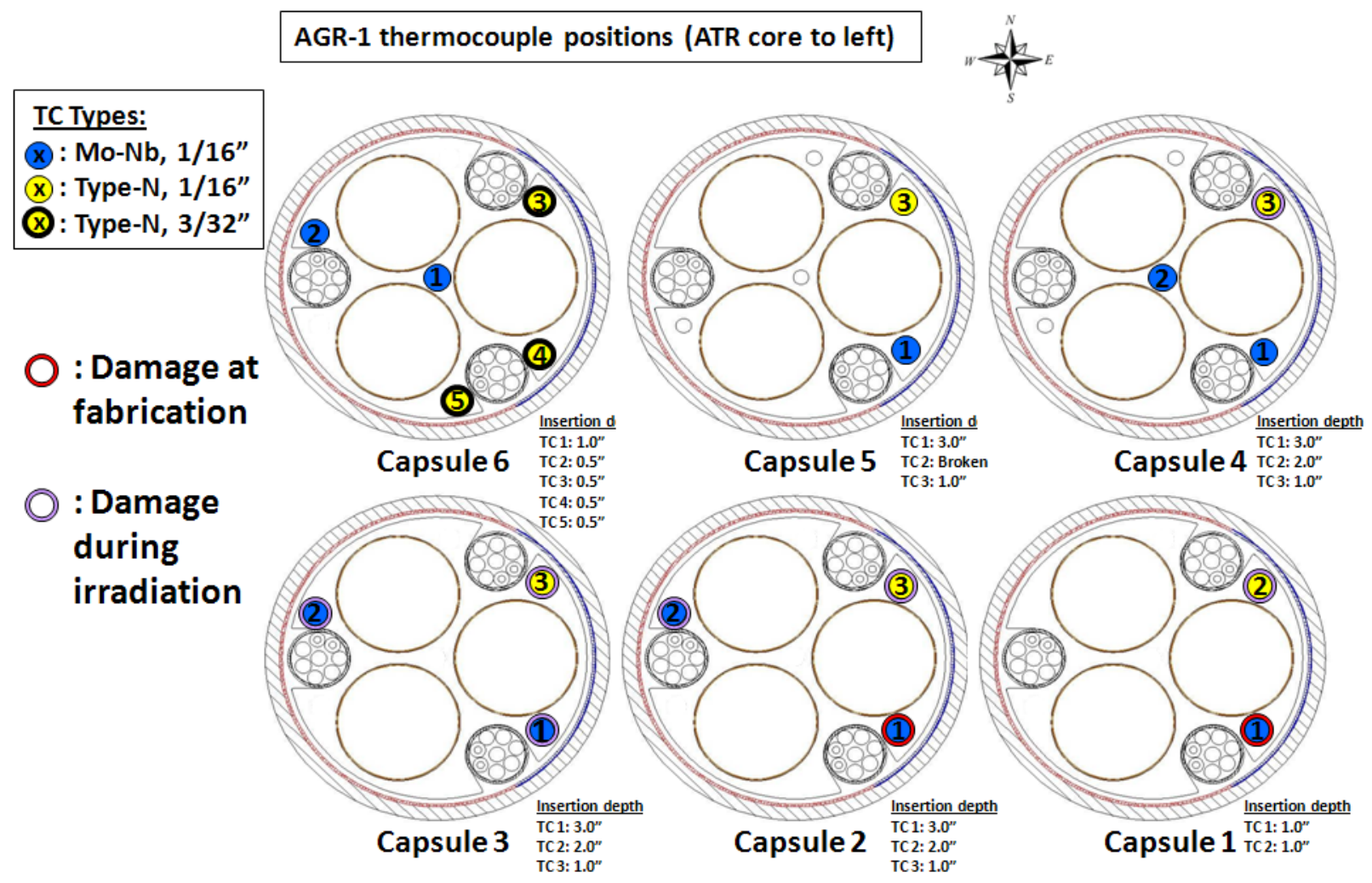

Figure 1. TC positions and types and their failure status for AGR-1 experiment.

\subsubsection{Simulation Data}

As part of the AGR-1 experiment, the as-run calculation of daily averaged temperatures (including target quantity fuel compact temperatures and graphite sample holder temperatures at all TC locations) from three-dimensional (3-D) finite element thermal models created for each capsule was performed because of the absence of direct fuel temperature measurement. These ABAQUS-based thermal models had been extensively verified (Ambrosek 2009; Hawkes 2011) but were not yet validated for AGR-1 test conditions. The input to these models were daily averaged component heat rates (mainly fuel and graphite) and fast fluence predicted from as-run physics depletion analysis (JMOCUP: coupled MCNP1.40 and ORIGEN2.2 [Sterbentz 2011]), gas mixture thermal conductivity estimated from the kinetic theory of gas as a function of temperature and helium and neon mole fractions, and fast fluence dependent thermal conductivity of fuel compacts and graphite components (Hawkes 2011). In addition to 
the test target quantity fuel compact temperature, the thermal model predicted temperatures at TC locations were outputs that served as important tools to calibrate and validate the simulation code and to assess the TC reading accuracy.

This section briefly describes the use of the simulation tools JMOCUP and ABAQUS. These descriptions are distilled from references developed by the respective modelers/analysts for physics and thermal simulations (Sterbentz 2011; Hawkes 2012). The focus is on the treatment of processes and elements that appear to influence comparative analysis of experimentally measured and numerically simulated data.

\subsubsection{JMOCUP Physics Analysis}

The primary physics parameters from the JMOCUP as-run daily depletion calculations are daily neutron fast fluence, fuel compact fission powers, and component heat rates for the entire AGR-1 irradiation period. The JMOCUP depletion calculation updates input data at the beginning of each time step, such as the actual ATR total core power, lobe powers, outer shim control cylinder (OSCC) positions, and neck shim withdrawals, along with the beginning-of-cycle ATR driver fuel-element loadings. Figure 3 presents daily averages of the ATR measured data needed in JMOCUP code for the AGR-1 experiment. The JMOCUP code, which modifies the existing MOCUP code to include AGR test parameters and requirements, has been carefully verified, but its validation will be limited to comparisons between calculated results and PIE measurement data by comparing compact/particle U-235 burnup and selected actinide and fission-product concentrations (Sterbentz 2011). The neutron induced activity of the flux wires embedded in the graphite holder measured in PIE will be converted to fluences with the appropriate neutron energy range to use as a benchmark for physics analyses (Maki 2009).

The Monte Carlo N-Particle (MCNP-1.40) code for each of the depletion cells calculates the cell neutron flux and nuclear reaction rate(s) for each specified isotope and nuclear reaction at each day-time step. The daily depletion time steps were required in part to account for the continuous adjustment of the OSCC during reactor operation. The AGR-1 test capsule was installed in the B-10 test facility, a test facility sandwiched between two OSCCs (E2 and E3). Rotational movement of these two cylinders can significantly impact the magnitude of the thermal neutron flux in the B-10 test facility and, hence, the fuel compact fission powers. The greatest increase to the thermal flux in a large-B position, such as B-10, is near the end of some ATR power cycles when the OSCCs are turned way out as shown on Figure 3. For example, in the end of cycle 139B, the four OSCCs in the northeast (NE) lobe (the dash green line in Frame 3 of Figure 2) rotated from 98 to 124 degrees. The impact of these large-angle end-of-cycle rotations is evident relative to the calculated capsule-power and TC-temperature measurements.

The JMOCUP depletion modeling includes the following assumptions (Sterbentz 2011):

1. The east-lobe source power is defined as the average of the NE, center (C), and southeast (SE) lobe powers; $\mathrm{E}=(\mathrm{NE}+\mathrm{C}+\mathrm{SE}) / 3$, and is used to normalize powers and fluxes in the B-10 test facility.

2. Fuel compacts were assumed to be constant at $600^{\circ} \mathrm{C}$ for ATR Cycle $138 \mathrm{~B}$ (first cycle) and $1200^{\circ} \mathrm{C}$ for the following 12 cycles.

3. High-temperature neutron cross-section data for actinides and fission products in the compacts were generated using primarily the Evaluated Nuclear Data File 6 (ENDF-6) (BNL 2009) data plus some ENDF-5 data at room temperature.

4. Compact materials are homogenized in the MCNP model, meaning that the TRISO particles and graphite binder matrix materials are assumed to be uniformly mixed. 


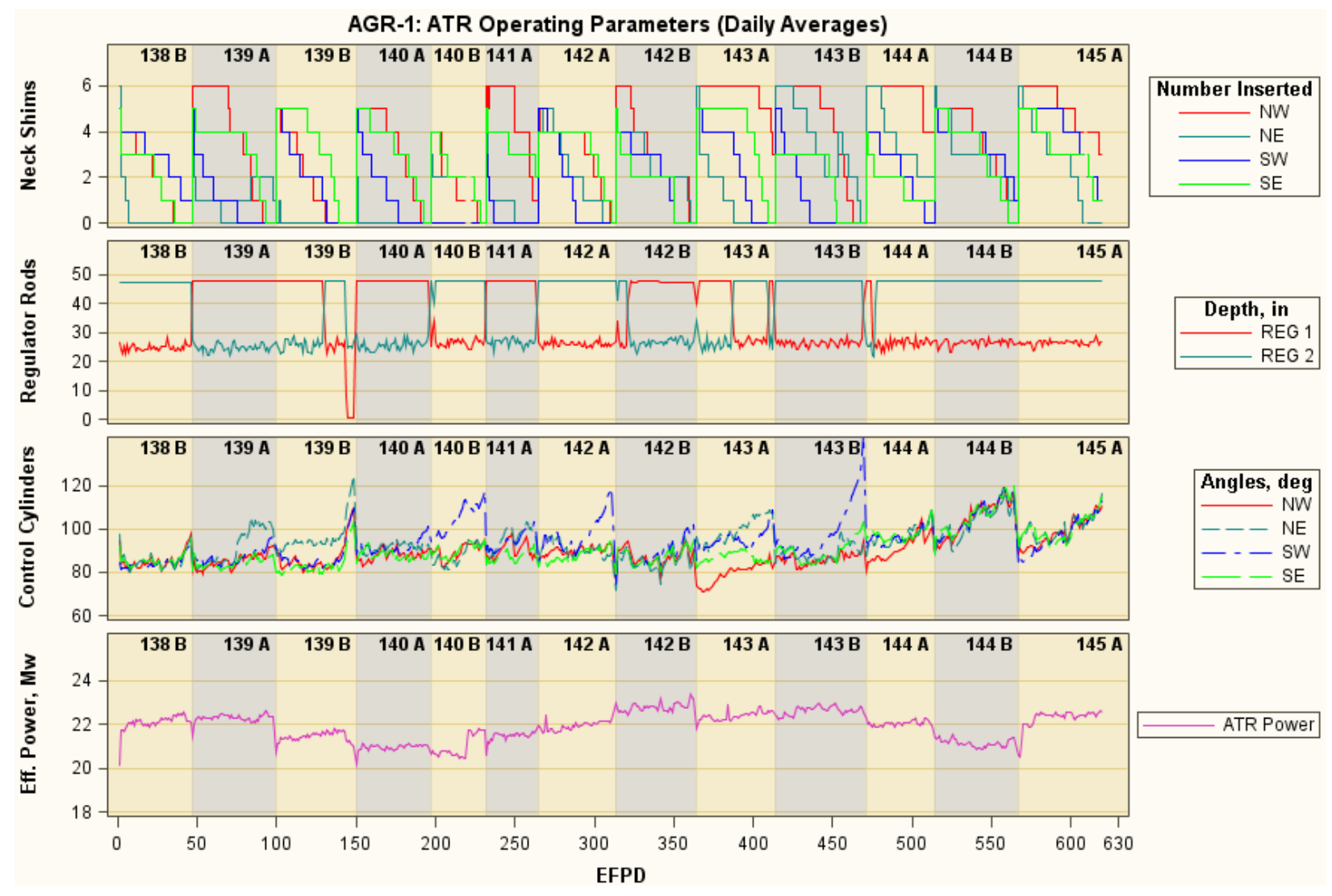

Figure 2. ATR operating parameters as function of AGR-1 EFPDs and ATR cycles.

\subsubsection{ABAQUS Thermal Analysis}

The ABAQUS based finite element thermal models are created for each capsule of the AGR-1 test to predict daily averages of fuel compact and TC temperatures for the entire irradiation period when the ATR core is at full power. The main time-series inputs to the model are daily component heat rates and fast fluences (mainly, fuel compacts and graphite sample holder) calculated from the as-run depletion analysis and daily gas compositions of helium-neon (neon fraction). The ABAQUS thermal model uses an $\sim 350,000$ eight-node hexahedral brick finite element mesh to estimate capsule temperature profiles. The validation of ABAQUS version 6.8-2 was performed and reported in (Hawkes 2012). It comprised of 10 thermal models validating different aspects of ABAQUS' heat transfer abilities. The maximum difference between ABAQUS calculated values and exact theoretical values is just under $2.0 \%$. However, uncertainty remains because of the lack of knowledge about phenomena such as heat- and neutron-induced changes in capsule control gas gaps.

The PIE metrology data of AGR-1 capsule components indicated that all fuel compacts unevenly shrank and the graphite sample holders unevenly swelled because of high boron addition to the graphite for middle Capsules 2, 3, 4, and 5 but shrank for Capsules 1 and 6 with less boron concentration. As the result, the control gap shrank for the four middle capsules and expanded for top and bottom capsules. Still, the gap between the graphite body and the fuel compact increased, offsetting somewhat the reduction in control gap in calculating fuel temperature. This behavior is being taken into account for the AGR-1 temperatures being calculated by assuming that the control gap is evenly and linearly reducing for middle four capsules and slightly increasing for top and bottom capsules over entire AGR-1 irradiation from initial actual hot gap size (subtracting thermal expansion of graphite holder) to the estimated end 
gap. Because there was no fuel particle failure in AGR-1, the R/B from the heavy metal contamination is very sensitive to temperature, and thus, is a measure of the temperature in each capsule. Therefore the end gap sizes were optimized by maximizing correlation between the temporal temperature predictions and the temporal R/B, indicating that the thermal model is capturing all of the important physical phenomena. The reduction of the end gap for four middle capsules could be as much as $50 \%$ of initial gap. These complicate the comparison between measurement and simulation.

Besides the gas-gap assumptions, the following factors and model assumptions may also contribute to predicted temperature uncertainties (Hawkes 2012):

1. Heat rates from components (excluding fuel compacts divided into two nodes) and fluences are spatially constant and vary only with time for each capsule.

2. Graphite and compact thermal conductivity varying with fluence and temperature is taken from legacy experiment correlations and scaled for AGR-1 material density.

3. Gas mixture thermal conductivity is determined by kinetic theory of gases using pure gas properties of helium and neon to determine mixture properties.

4. Heat transfer through gas is by conduction and radiation only-no advection because of the very low flow rate of gas.

5. Radiation heat transfer occurs from the graphite holder to the stainless-steel retainer, graphite holder to thru-tubes, and thru-tubes to stainless-steel retainer. An emissivity of 1.0 and 0.99 were assumed for these surfaces in order to closely match the TC readings during earlier cycles, when TC performance is believed to be more reliable.

6. There is no axial heat conduction from one capsule to the next.

The example of fuel temperature distribution in Capsule 4 during Cycle 141A is presented in Figure 3 (Hawkes 2011). Figure 3 shows a cutaway view of the three fuel compact stacks. Temperatures range from $802^{\circ} \mathrm{C}$ to a maximum of $1256^{\circ} \mathrm{C}$. According to this distribution, the highest temperature is at the capsule center and temperature is decreasing for locations departing from the center both radially and axially. In general, temperatures are higher for Stack 1 and 3 located in the capsule western side directly facing the ATR core and lower for Stack 2 located in the capsule eastern side and shielded from the ATR core. 


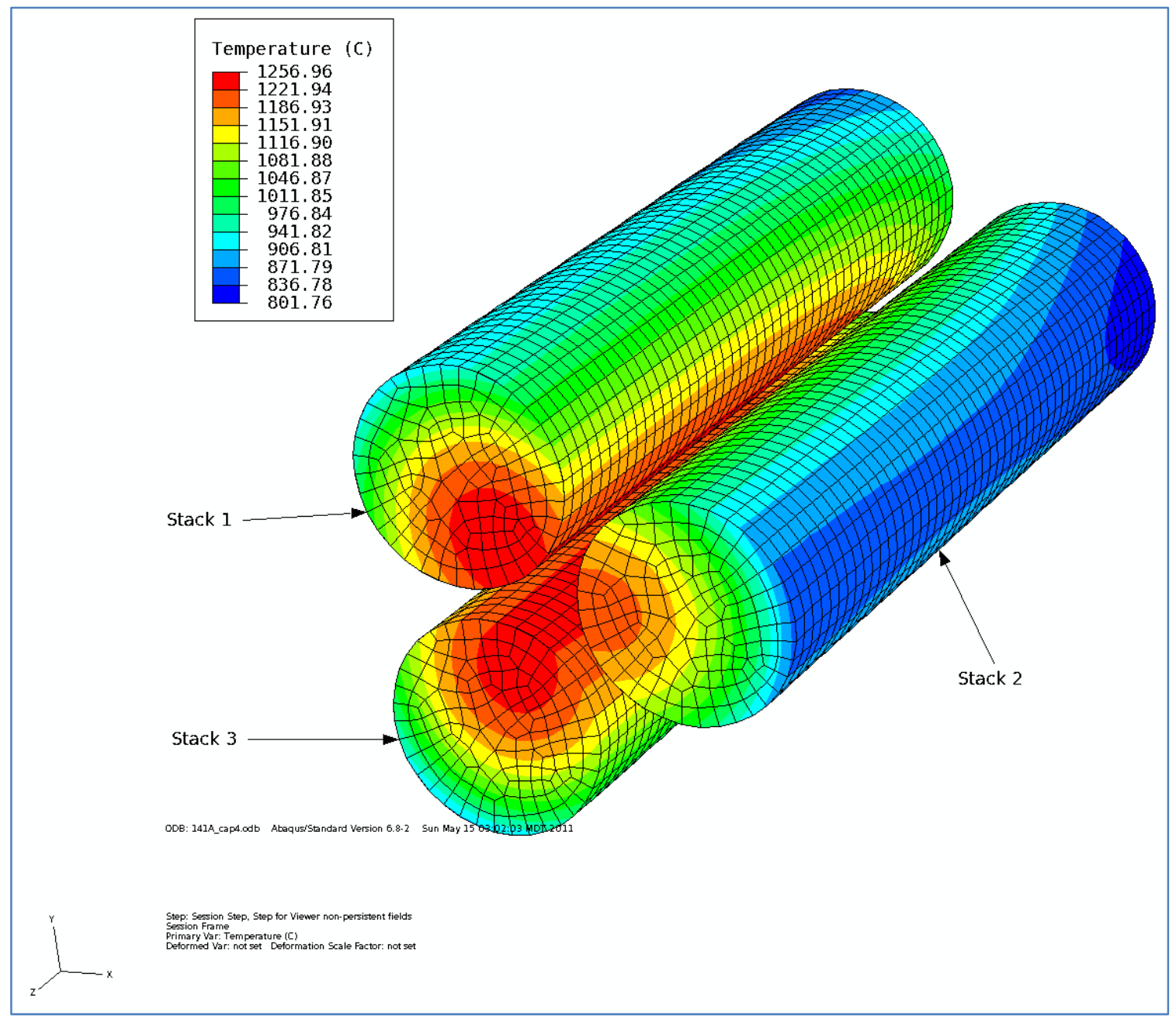

Figure 3. Temperature $\left({ }^{\circ} \mathrm{C}\right)$ contour plot of cutaway view of three fuel stacks during cycle $141 \mathrm{~A}$.

\subsubsection{Data Trends}

The graphical summary containing plots of capsule irradiation condition parameters as a function of time is used for data trending and anomaly identification. The time is expressed by AGR-1 EFPDs, which are accrued as time passes in the experiment based on the ATR full-power level and excludes all ATR down time periods. In addition to EFPD, the horizontal axis also contains corresponding ATR cycles, presenting parameters behavior within each cycle. The graphical summary for each capsule (see example in Figure 4) contains six frames: Frame 1 shows actual TC temperatures; Frame 2 shows neon fraction of capsule temperature control gas mixture; Frame 3 shows calculated fuel fission power density and graphite holder power density (in w/cc); Frame 4 shows TC residuals (differences between measured and simulated temperatures for each TC); Frame 5 shows simulated capsule average, maximum, and minimum fuel temperatures; and Frame 6 shows R/Bs of three fission product radionuclides. The neon fraction and fuel and graphite powers are included because they are directly related to capsule temperature. The fission product $\mathrm{R} / \mathrm{Bs}$ of three main isotopes $(\mathrm{Kr}-85 \mathrm{~m}, \mathrm{Kr}-88$, and $\mathrm{Xe}-135)$ are included because of their close relationship to temperature. Combining these relevant parameters together helps explain observed data trends. 
Consistent data trends and anomalies suggest either potential equipment failure or the physics-based models' inability to completely describe the complex processes in the AGR test capsules. As an example, the graphical summary for Capsule 6 (Figure 4) depicts the following significant trends:

- The Neon fraction plot in Frame 2 shows the effect of helium leaking from lead-out gas flow into the capsule gas gap before May 29, 2009, reducing the neon fraction (neon fraction is about 0.8 when there was only neon in gas mixture).

- The scatterplots of differences between measured and simulated temperature (TC residuals) for five TCs in Frame 4 are level at about negative $120^{\circ} \mathrm{C}$ of TCs during the entire AGR-1 irradiation period, suggesting possible bias in simulation. In this frame, the upward trend of TC residuals within each ATR cycle for all TCs is also apparent. These consistent trends suggest aspects of the simulation models (physics and/or thermal) that could be improved.

- The instantaneous peak fuel temperature plotted in frame 5 slightly exceeds the specification limit during ATR Cycle 143A. The fuel temperature specifications for the AGR-1 test are: (1) the instantaneous peak fuel temperature is less than $1400^{\circ} \mathrm{C}$ to minimize over heating of test fuel, or (2) the time-average volume-average temperature is within the range of $1150+30 /-75^{\circ} \mathrm{C}$, ensuring a sufficiently high temperature without exceeding the peak fuel temperature limits (Maki 2004).

- The upward trend of R/B data plotted in Frame 6 during the entire irradiation period, especially when calculated fuel temperatures (Frame 5) were decreasing toward the end of the experiment, may indicate that the thermal model failed to predict an increase in fuel temperature. 


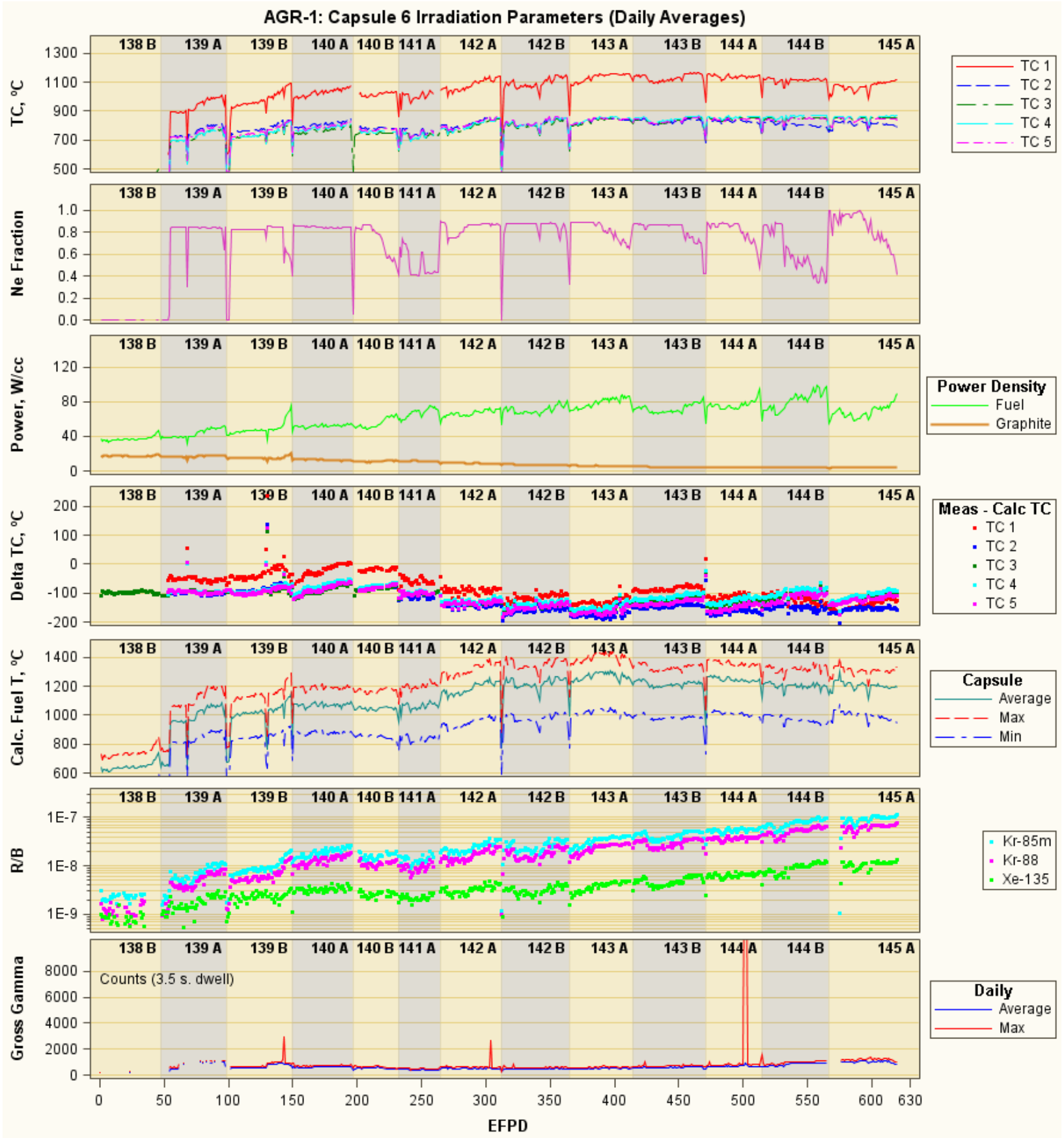

Figure 4. Graphical summary of AGR-1 irradiation data for Capsule 6.

\subsection{Statistical Analysis Methods for AGR Data Qualification}

This subsection describes the data qualification process and statistical analysis methods implemented in the NDMAS. Analysis of the NGNP fuel irradiation data collected from the AGR test campaign is the main focus of this report. Data users and data generators determine the data that are needed for their application and the requirements the data must meet. In the NGNP Program, the data generated will be used to support the VHTR design and licensing and must therefore comply with the collection criteria of the American Society of Mechanical Engineers "Quality Assurance Program Requirements for Nuclear 
Facility Applications" (NQA-1; INL 2010). Thus, all quality-affecting activities performed by INL management and operations in the Very High Temperature Reactor Technology Development Office follow the "Very High Temperature Reactor Technology Development Office Quality Assurance Program Plan" (INL 2009a). Both measurement and simulation data generated from AGR test campaign belong to this category.

TC failure for the AGR experiments has different manifestations - some are easy to recognize when the readings are clearly out of the expected measurement range or simply drop to zero (signal loss). Other malfunctions are harder to identify, for instance, when the TC readings are still within the valid range, but have a certain bias (drift) or have formed a virtual junction somewhere in the test section or along the bundle cable. In fact, the virtual junction failure of two TCs in the AGR-1 test was discovered by a neon gas test at the beginning of irradiation. This test varied neon fraction of the control gas mixture in one capsule (test capsule) at a time to see how TCs in other capsules responded to the gas composition change. When a TC in the other capsule responds to thermal condition changes in the test capsule, this TC might form a virtual junction with the test capsule, thus its readings are no longer accurate. However, this type of testing was conducted only once during the irradiation campaign. Thus, it is highly desirable to have other methods such as statistical analysis for identification of such deceptive modes of instrument failure. Furthermore, some data from the deteriorated TCs may still be useful when the possible bias can be quantified and accounted for. The data trend of these TCs could still be used for test control or code validation. Early identification of all TC failure types is an important task for the entire fuel performance and qualification campaign.

\subsubsection{Data Qualification and Analysis in NDMAS}

The NDMAS data qualification activity supports the VHTR Quality Assurance Program Plan goal as shown in Figure 5. The independent review and testing of data accuracy by NDMAS verify that the data collected within an NQA-1 program have met the requirements for the designated planned data collection activity. At a minimum, qualified data in NDMAS are required to meet the following requirements (INL 2009b):

- Data must be correctly captured as being identical to the original data generated by the data collection programs

- Data must be an accurate representation of the system or object being measured or modeled

- Data must have been collected under an NQA-1 or equivalent approved Quality Assurance program.

- Data review and validation as elements of data qualification are the main focus of NDMAS.

As a result of the data review, data qualifiers or flags are applied to alert the end-user to potential quality problems that may impact the usability of the data (e.g., requirements were not met). However, measurement uncertainty in the data review process is not evaluated in a quantitative manner, which is why data review or validation is only a "first-step process" for the assessment of data usability. The full data usability assessment is more complex and multifaceted than data review or validation, requiring greater understanding of both underlying physical processes in the experiment and conditions of data usage. The review and testing of data can lead to one of three disposition states: qualified, trend, or failed. Qualified data meet all requirements. Trend data have minor biases but are still useful to the program. Failed data are deemed unusable because of gross failure in meeting the requirements. Testing and review records are maintained in the NDMAS system to provide an auditable trail of data qualification. The qualification status of each data record in the system is identified to support the quality-cognizant and controlled use of the data. 


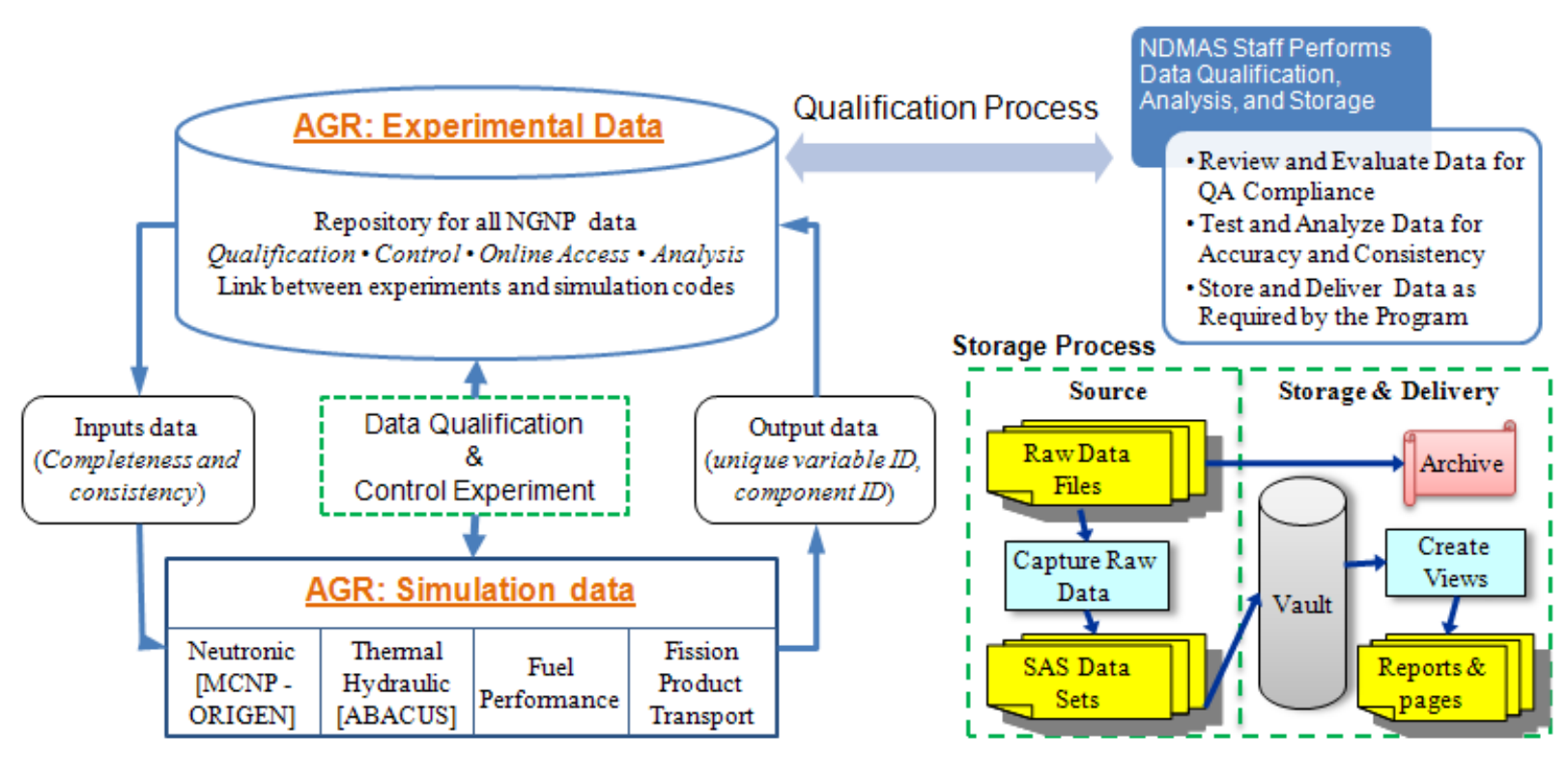

Figure 5. NDMAS data qualification and analysis to support AGR tests.

Conventional techniques such as capture and range checks can be used in data qualification to remove apparent and gross failures of the measuring instruments (e.g., TCs). However, data anomalies because of deteriorated instruments (e.g., drift or junction) are more subtle, requiring more sophisticated techniques to detect. Notably, variations in the AGR test data are caused not only by measurement device failures, but also by changes in the experimental conditions (both planned and unexpected). Statistical techniques (e.g., control charting, correlation analysis, and summary statistics) can be used to identify and flag the "less obvious" anomalies for further investigation. As a rule, decisions validating or invalidating suspect data and its reliability for further use requires application of both statistical and engineering concepts. The ultimate decision about a data anomaly is made by subject matter experts (e.g., the NGNP Project Data Review Committee), which review all statistically identified out-of-bound events for possible scientifically defensible explanations in order to specify these data as qualified. Otherwise, these data must be flagged as trend or failed.

For traceability, the resolution must then be documented in NDMAS, including identification, analysis, qualification decision, and its justification.

\subsubsection{Statistical Methods for Data Accuracy Monitoring}

Statistical techniques used in data qualification range from calculation of simple statistics to more advanced modeling and analysis. The traditional analysis involves determining the data statistics (e.g., population mean and standard variation) or the relationship (e.g., correlation and regression function) between random variables of interest using the past data (baseline or training period). These are then used to monitor the future data (monitoring period) and identify instances when measured data cross the established statistical bounds. Early identification of data anomalies by the statistical models will help timely collection of onsite information about the factors (which might otherwise be lost or forgotten) that later prove critical for modeling and code validation.

NDMAS primary data qualification functions include statistical analysis modules built on the Statistical Analysis System (SAS) Enterprise Business Intelligence software (SAS 2007). To support data qualification of the AGR-1 TC readings, three statistical analysis methods were selected and applied to provide evidence of TC failures: control charting (CC), correlation analysis (CA), and regression analysis 
(RA). These methods use properties of TC data and their relationships specific to the AGR test to identify different modes of TC failures. CA takes advantage of the fact that correlation between readings of TCs in the same capsule (within-capsule) is stronger than between readings of TCs in different capsules (intercapsule). This allows for the monitoring of changes in correlation coefficient statistics, which is potentially useful in discerning TC virtual junction failure. RA uses the regression relationship between TCs and other relevant quantities to monitor for departure of future TC readings from regression predicted uncertainty bounds - potentially useful in identifying TC drift failure. CC uses a graphical display to monitor for departure of measurements from established statistical bounds, indicating either instrument failure or change in the test condition. More importantly, the three methods (CC, CA, and RA) together provide a complementary capability in TC failure identification. The TC accuracy monitoring summary is presented in Figure 6. The detailed methodology of these statistical methods is presented in Pham and Einerson (2010).

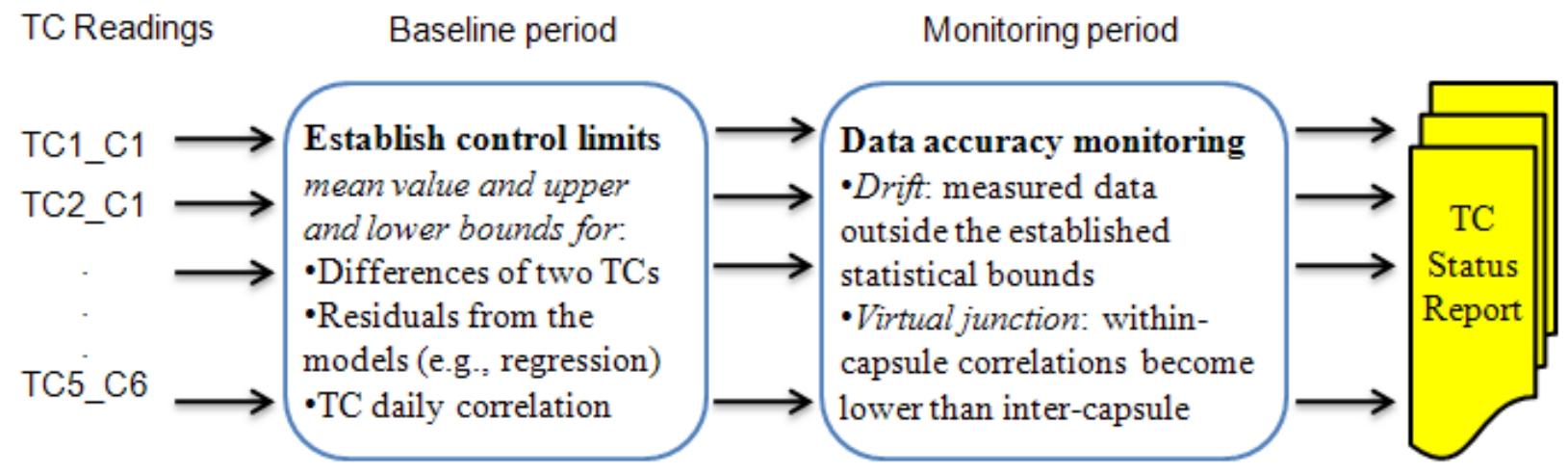

Figure 6. Thermocouple measured data accuracy monitoring in AGR experiment.

The present work exercises the idea that, while recognizing uncertainties inherent in physics and thermal simulations of the AGR-1 test, results of the numerical simulations can be used in combination with the statistical analysis methods to further improve measured data qualification. The simulated temperature changes result from the same physical processes in capsules, and therefore could be used as references to refine the identified abnormalities. For example, when the control chart of differences between TC pair readings show out-of-control instances, indicating either drift failure of at least one TC or change in experiment condition, the simulation data are used to assess if and which TC is actually failed. Hence, simulation data will help reduce measurement uncertainty. 


\section{QUALIFICATION OF AGR-1 TC DATA}

All TCs in Capsules 1, 2, and 3 and TC3 in Capsule 4 were failed by NGNP experts at various times during the AGR-1 test (Section 1.2.1). The experts are experiment performers, modelers and data analysts. The failed data were subsequently flagged as disqualified in the NDMAS database and so were removed from all data analysis here. The remaining TCs (namely, TC1 and TC2 in Capsule 4, TC1 and TC3 in Capsule 5, and TC1 through TC5 in Capsule 6) have qualified measurements for the entire irradiation. These qualified TC measurements are subject to more sophisticated accuracy tests for consistency and trend for TC performance assessment. Since there was no indication of TC virtual junction failure, this section focuses on detection of TC drift failure.

\subsection{TC Drift Detection Method}

The term TC drift refers to the differences in TC readings over time that are the result of a malfunctioning TC rather than changes in experimental conditions. TC drift monitoring is based on measurement and simulation data of a TC pair in the same capsule and looks at four panels of plots as functions of time as shown in Figures 8 through 14 (below): control charts of TC pair temperature differences for both measurement and simulation; TC residuals (measured minus simulated); daily correlation coefficients; and actual measured and simulated TC values. These monitoring plots provide complementary indications of potential TC drift and help define the failure mode. The TC drift criteria are determined as follows:

1. Control charts of TC pair temperature differences: For a stable TC pair located in thermally similar locations, the temperature differences in the monitoring period should stay within the established control limits of three standard deviations around the mean value. When consistently out-of-control instances in a monitoring period are apparent, two scenarios should be considered:

a. The measured TC differences follow the simulated TC differences (the two plots are parallel), and the out-of-control instances are justified and the TC pair is deemed stable.

b. Otherwise, at least one of the TCs in the pair might be drifting.

2. TC residuals: The TC is deemed stable relative to simulation when its residuals, as a function of time, lie around a horizontal line. Therefore, a consistent slope of TC residuals indicates a TC drift (either downward or upward depending on slope direction).

3. Daily correlation coefficients: The correlation coefficients between within-capsule TC pairs should be close to 1 . Therefore, decreasing daily correlation coefficients indicate that at least one TC of the subject TC pair is deteriorating.

4. Actual measured and simulated TC data plots: These plots are used to confirm the drift indication identified in items (1) to (3) by the departure of the actual readings of a drifting TC from being parallel to both its simulation and its reading of the other TC.

\subsection{AGR-1 TCs Quality Assessment}

The drift monitoring plots based on measured and simulated within-capsule TC pair comparisons shown in Figures 7 to 14 are used to assess the drift failure for all TCs in the AGR-1 experiment. All of the data used in this analysis are filtered to time spans when the ATR effective power (the average of the power in the center and east lobes) was at least $20 \mathrm{MW}$ to exclude the start-up and shout-down transient period because of high uncertainty in simulation data. The TC drift indications for all TCs in the AGR-1 experiment are discussed below. 


\subsubsection{Capsule $6\left(\mathrm{TC} 3,4\right.$, and 5 are stable, but biased down by more than $100^{\circ} \mathrm{C}$; TC1 and 2 are drifted down during the last two cycles)}

Capsule 6 (top left capsule in Figure 1) has five surviving TCs. TC1 and TC2 are a molybdenumniobium type TC with thin diameter wire (1/16 in.). TC1 is located radially in the capsule center at a 1-in. depth in the graphite sample holder and experienced the highest temperature; TC2 was positioned in the western periphery (sandwiched between the hotter fuel Stacks 1 and 3 Section 1.2.2.2) at 0.5-in. depth and experienced lower temperatures than TC1, but higher than TC3, TC4, and TC5, which are located in the eastern periphery (shielded away from the ATR core) at 0.5 -in. depth. All three TCs $(3,4$, and 5) are Type-N with thicker diameter wire (3/32 in.) and are located in similar positions, so their readings should be close to one another. This order of TC reading levels remained during most cycles of the AGR-1 irradiation period (Frame 1 of Figure 2). The thermal model also predicted the same temperature order at these TC locations.

\subsubsection{TC3, TC4, and TC5}

TC3, TC4, and TC5 are stable. First, the same close-to-perfect normal distribution of the TC residuals histogram for TC3, TC4, and TC5 in Figure 7 indicates that all three TC readings are consistent with their simulation with about 110 to $120^{\circ} \mathrm{C}$ bias. Second, the TC drift monitoring plots for the two TC pairs presented in the Figure 8 (TC3-4 pair on the top row and TC3-5 on the bottom row) also provide adequate evidence that TC3, TC4, and TC5 responded similarly relative to each other and to their simulation throughout the entire AGR-1 irradiation period:

- Control charts of TC pair temperature differences (Frame 1): For TC3-4 pair (top row image), both measured (red dots) and simulated (purple dots) TC differences are close to each other and stay within the tight control limit band of $250 \mathrm{C}$. On other hand, the out-of-control instances for measured differences of the TC3-5 pair (bottom row image) toward the end of the experiment were justified by the same behavior of simulated TC differences.

- TC residuals (Frame 2): The residual plots of all three TCs closely follow each other and consistently stay at the same level of more than $-100^{\circ} \mathrm{C}$ during the entire irradiation period ($117^{\circ} \mathrm{C}$ for $\mathrm{TC} 3,-110^{\circ} \mathrm{C}$ for $\mathrm{TC} 4$, and $-122^{\circ} \mathrm{C}$ for TC5). This means the simulated TCs had a bias of 110 to $120^{\circ} \mathrm{C}$ relative to $\mathrm{TC} 3, \mathrm{TC} 4$, and $\mathrm{TC} 5$ readings.

- Correlation coefficients (Frame 3): The high correlation coefficients for both TC pairs throughout the whole experiment indicate the consistency of these TC responses to the capsule thermal condition.

- Actual measured and simulated TC plots (Frame 4): Both plots for measured and simulated temperatures of two pairs of TCs, TC3-4 and TC3-5, are very consistent with each other and their simulation for entire irradiation period.

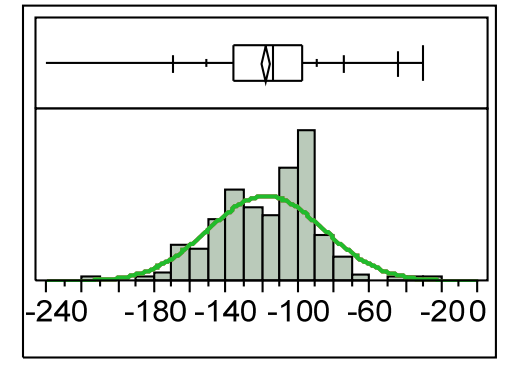

TC 3: $116.7 \pm 25.9^{\circ} \mathrm{C}$

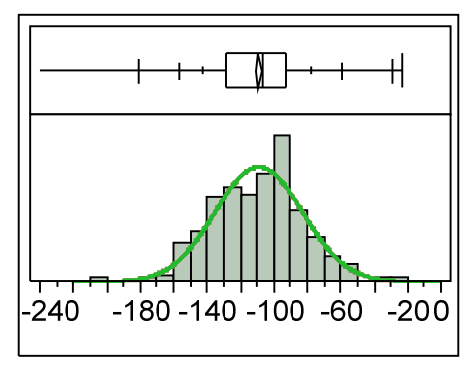

TC 4: $109.6 \pm 26.2^{\circ} \mathrm{C}$

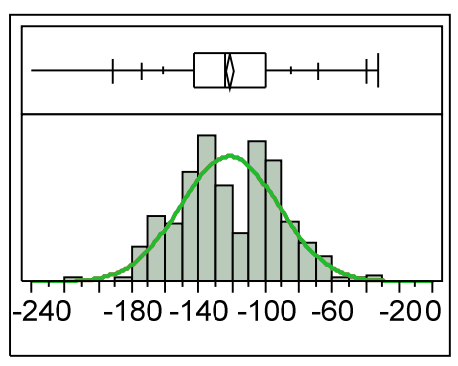

TC 5: $122 \pm 29.4^{\circ} \mathrm{C}$

Figure 7. Histograms of temperature residuals of TC3, TC4, and TC5 of Capsule 6 

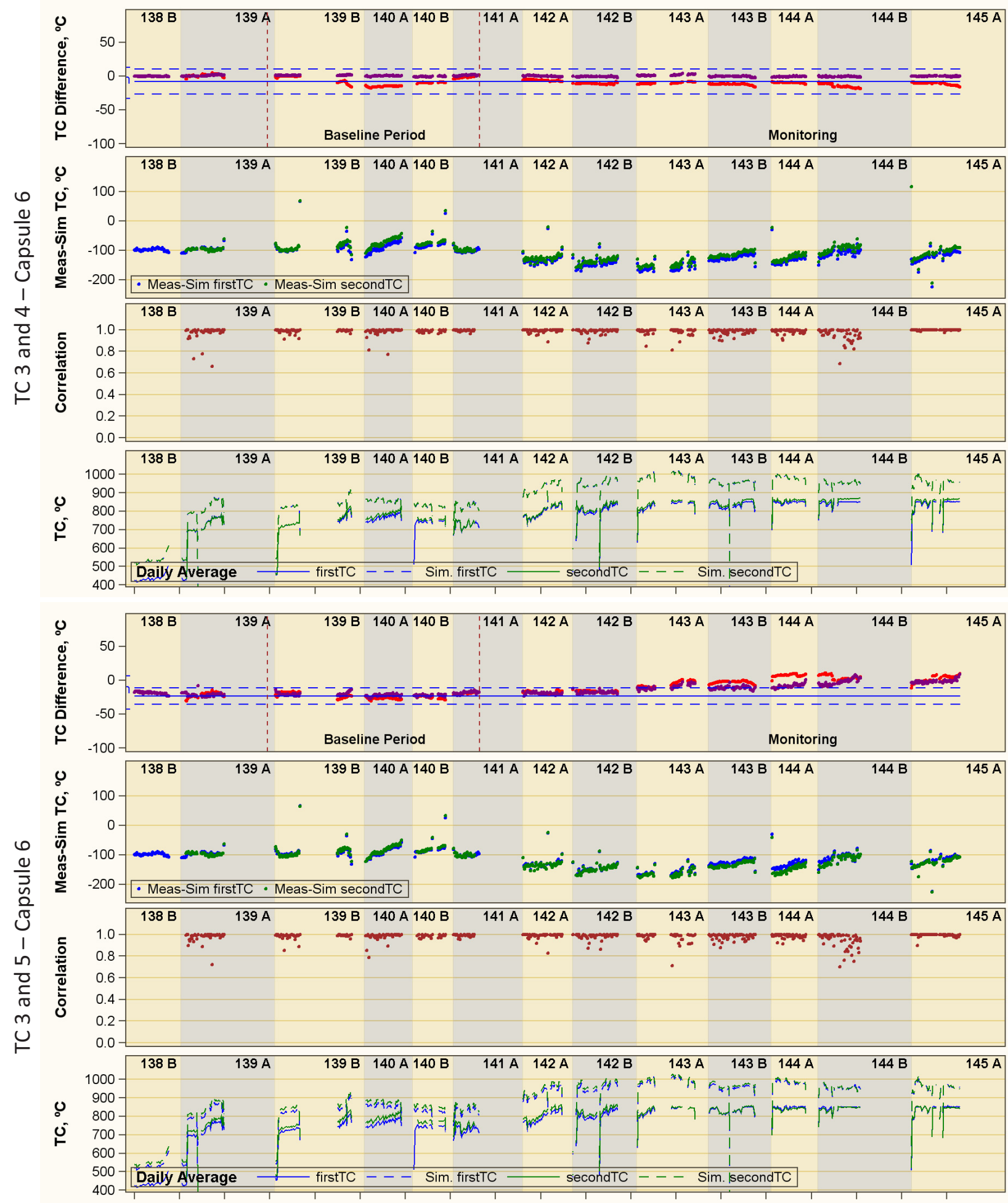

29Dec 24Feb 22Apr 18Jun 14Aug 100ct 06Dec 01Feb 29Mar 25May 21Jul 16Sep 12Nov 08Jan 06Mar 02May 28Jun 24Aug 200ct $20062007 \quad 2008 \quad 2009$

Figure 8. The drift monitoring for TC $3 \& 4$ and TC $3 \& 5$ pairs of Capsule 6. 


\subsubsection{TC1}

TC1 slightly drifted down as shown in the drift monitoring plots for the pair of TC1 and stable TC3 (TC1-3 pair in the top row of Figure 9) as follows:

- Control charts of TC pair temperature differences (Frame 1): the measured differences (red dots) are higher than the simulated differences (purple dots) by $\sim 50$ degrees at the beginning of the irradiation, and then $\sim 20^{\circ} \mathrm{C}$ lower at the end of the experiment resulting in a possible TC1 drift of $\sim 70^{\circ} \mathrm{C}$ given that TC3 is stable. Even though there are no out-of-control instances for measured TC1 and TC3 differences in the monitoring period, the crossing of measured and simulated TC differences (red and purple dotted lines) indicates that TC1 might be drifting relative to the stable TC3 and the TC1 simulation.

- $\quad$ TC residuals (Frame 2): TC1 residuals (blue dots) are around $-50^{\circ} \mathrm{C}$ and parallel to $\mathrm{TC} 3$ residual plots until the end of Cycle $140 \mathrm{~B}$, then gradually dropped to about $-120^{\circ} \mathrm{C}$ toward the end of the experiment resulting in about $70^{\circ} \mathrm{C}$ drift.

- Correlation coefficients (Frame 3): the decreasing correlation coefficients toward the end of the experiment (the brown dots) indicate that TC1 and TC3 don't respond to the capsule thermal condition the same way as in earlier cycles.

- Actual TC plots (Frame 4): measured TC1 (continuous blue line) and simulated (dashed blue line) temperature plots are departing further from one another after Cycle 140B.

\subsubsection{TC2}

TC2 slightly drifted down as shown in the TC drift monitoring plots for the pair of TC2 and stable TC3 presented in the bottom row of Figure 9 as follows:

- Control charts of TC pair temperature differences (Frame 1): The measured differences (red dots) were below the control limits and became increasingly lower than simulated difference (purple dots) after cycle 142A. Even though the simulated differences were slightly down after cycle $142 \mathrm{~A}$, the measured differences were down at a much greater rate, especially during the last two cycles.

- $\quad$ TC residuals (Frame 2): TC2 residuals (blue dots) are in line with TC3 differences (green dots) until Cycle 143A, and then gradually decrease. By the end of the experiment, TC2 residuals were $50^{\circ} \mathrm{C}$ lower than $\mathrm{TC} 3$ residuals.

- Correlation coefficients (Frame 3): The decrease in correlation coefficients toward the end of the experiment (the brown dots are scattering lower, especially during the last two cycles) also indicates deterioration of TC2 relative to the stable TC3.

- Actual TC plots (Frame 4): TC2 readings (blue line) dropped lower than TC3 during the last three cycles (green line), while simulated TC2 values (dashed blue line) and TC3 values (dashed green line) are much closer to each other. 


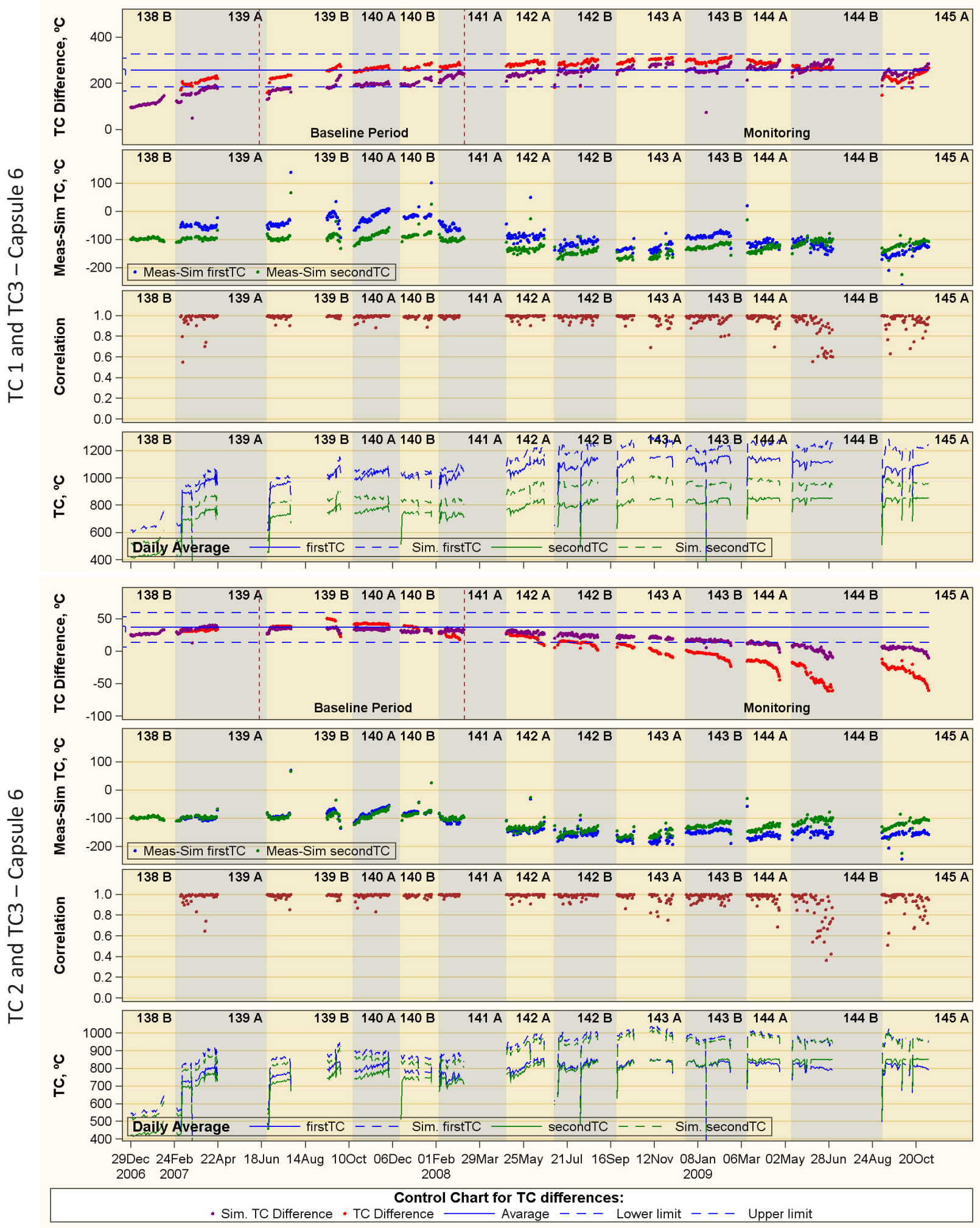

Figure 9. The drift monitoring for TC $1 \& 3$ and TC $2 \& 3$ pairs of Capsule 6. 


\subsubsection{Capsule 5 (TC1 drifted upward by $200^{\circ} \mathrm{C}$ and $\mathrm{TC} 3$ is stable with wide variation)}

Capsule 5 has two TCs with thin wire 1/16 in. in diameter. TC1 is molybdenum-niobium type and TC3 is type-N. They both are located in the capsule eastern periphery and shielded from the ATR core. They were located at the same distance from the capsule center axially. TC1 was inserted in 3.0 in. depth and TC3 was inserted in 1.0 in. depth in the graphite sample holder. Therefore, their readings are expected to be close to each other as shown by the model predicted TC1 and TC3 (dashed blue and green lines in Frame 4 of Figure 10). However, the drift monitoring for the TC1-3 pair in Capsule 5 presented in Figure 10 found that the TC3 readings are up to $100^{\circ} \mathrm{C}$ higher than the simulation for the first half of the AGR-1 experiment and up to $100^{\circ} \mathrm{C}$ lower during the second half. On the other hand, the TC1 readings are drifting upward by $200^{\circ} \mathrm{C}$ during entire AGR-1 irradiation period. This drift detection case most clearly demonstrates the essential of having the simulation data as part of the TC performance assessment.

- Control charts of TC pair temperature differences (Frame 1): This figure shows the clear departure of the measured TC difference plot (red dots) from the mostly horizontal position of the simulated difference plots (purple dots) right from the beginning of the experiment. The strong upward trend of measured TC differences over time, while the simulated differences were slightly down, clearly indicates that at least one TC of the pair drifted, but it does not identify which TC.

- $\quad$ TC residuals (Frame 2): Since the TC1 residuals (blue dots) are increasing upward, from $50^{\circ} \mathrm{C}$ to about $200^{\circ} \mathrm{C}$, during entire AGR-1 irradiation period, TC1 can be considered drifted relative to the simulation. On the contrary, TC3 residuals (green dots) were increasing gradually from $0^{\circ} \mathrm{C}$ at the AGR-1 beginning to about $100^{\circ} \mathrm{C}$ at the end of the Cycle 140B. Then the residuals decreased to negative $100^{\circ} \mathrm{C}$ by the end of AGR-1. As a result, TC3 is considered to be relatively stable in relation to simulation, but with wide variation. This is also consistent with the amount of the upward trend in measured TC1 and TC3 differences plotted in Frame 1.

- Correlation coefficients (Frame 3): The fact that the daily correlation coefficients are still high for the TC1-3 pair means that both TCs still respond to the capsule thermal condition, but its calibration changed. Therefore, in spite of the drift, the TC1 readings are still useful for capsule temperature control if the TC bias is properly quantified and accounted for.

- Actual TC plots (Frame 4): The clear upward departure of the TC1 readings (solid blue line) from TC3 readings (solid green line) over the course of the experiment, while the simulated temperatures at TC1 and TC3 locations (dash blue and green lines) remained close to each other, confirms that TC1 drifted.

The histograms of temperature residuals for TC1 and TC3 of Capsule 5 in Figure 11 also lead to the same conclusion that TC3 is stable and TC1 drifted upward. The histogram of TC3 residuals (on the right) has the mean value near zero $\left(-10^{\circ} \mathrm{C}\right)$, which is a major criterion for an excellent agreement between measurement and simulation of TC1 readings. However, the TC3 residuals histogram had two distinct clusters: the first is a cluster around $50^{\circ} \mathrm{C}$, representing the reading before Cycle $142 \mathrm{~A}$, and the second is a cluster around $-50^{\circ} \mathrm{C}$, representing $\mathrm{TC}$ readings after that cycle. This split in the histogram reflected the shift of $100^{\circ} \mathrm{C}$ in TC3 readings at the end of Cycle 141A. On other hand, the TC1 residuals histogram (on the left) is shifted to the right instead of centered on zero, indicating that TC1 readings were biased up compared with its simulation. This histogram helps illustrate a potential bias in TC1 readings relative to simulation. 


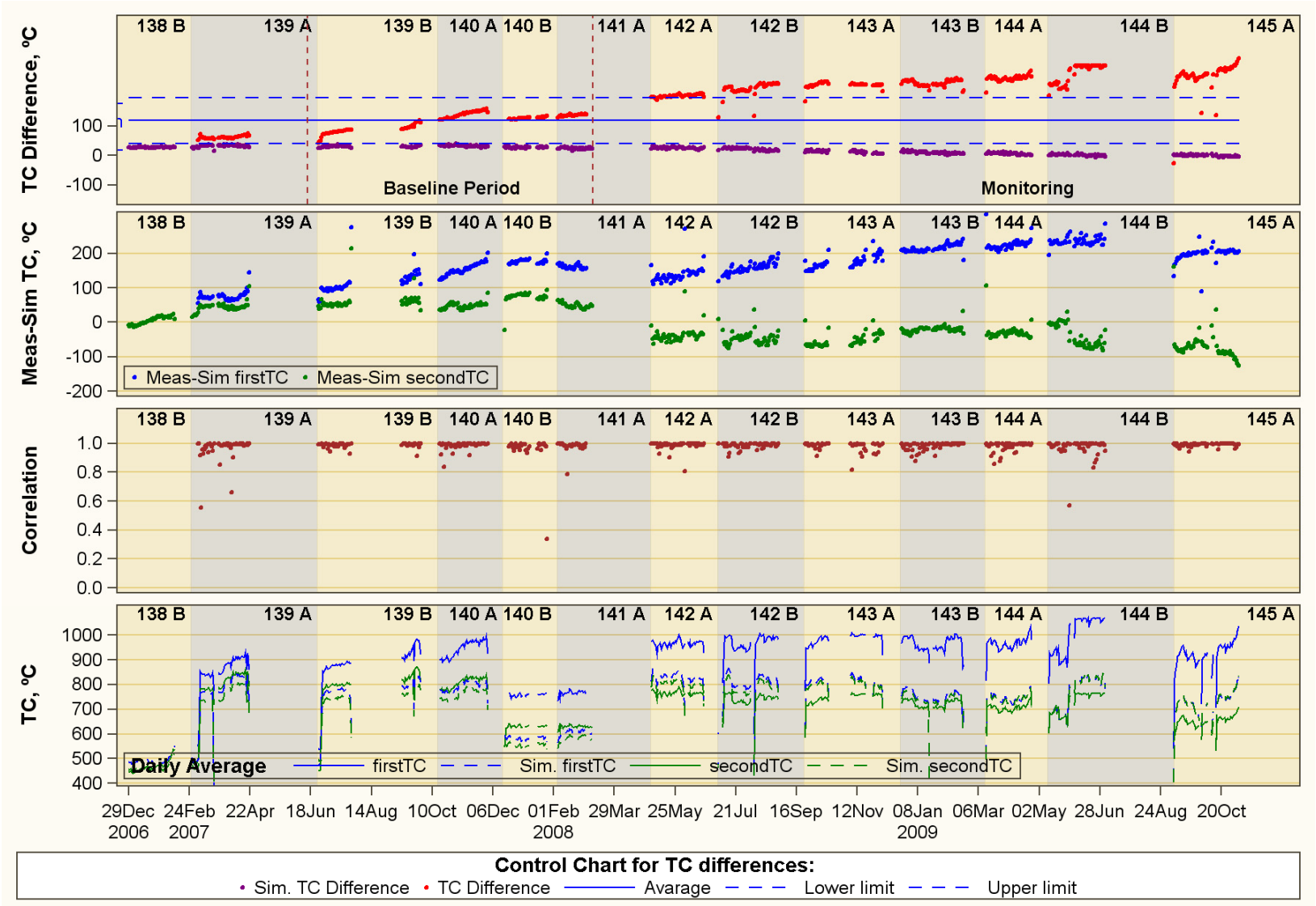

Figure 10. The drift monitoring for TC1 and TC3 pair of capsule 5.

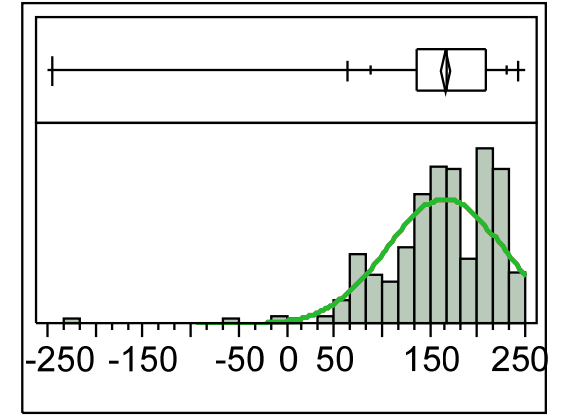

TC 1: $164.9 \pm 61^{\circ} \mathrm{C}$

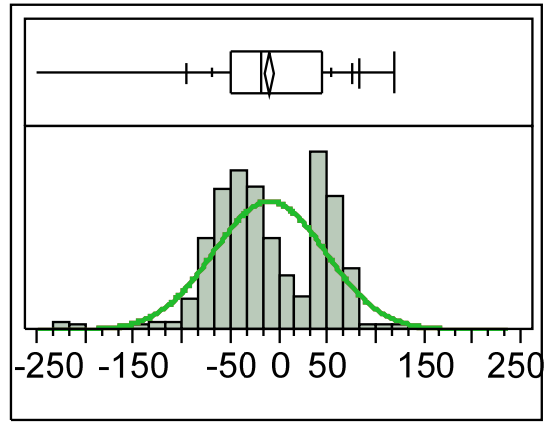

TC 3: $-10.6 \pm 57.7^{\circ} \mathrm{C}$

Figure 11. Histograms of simulated minus measured for TC1 and TC3 in capsule 5.

\subsubsection{Capsule 4 (TC1 and TC3 were stable and TC2 drifted down by more than $\left.100^{\circ} \mathrm{C}\right)$}

Capsule 4 has three thin wire TCs (1/16 in. diameter), TC1 and TC2 are molybdenum-niobium type and TC3 is Type-N. TC2 is positioned in the capsule centerline and inserted at a 2 in. depth in the graphite sample holder located in the hottest region of the graphite sample holder. TC1 and TC3 are located in thermally similar positions in Capsule 4 (in eastern periphery shielded from ATR core and 
inserted at $3 \mathrm{in.}$ and $1 \mathrm{in}$. depth respectively in the graphite sample holder so they are at equal distant from the capsule center). All readings of TC1 and TC2 are qualified in NDMAS for the entire AGR-1 experiment and TC3 was failed on October 16, 2008, during Cycle 143A by program experts. TC3 readings were recorded from the beginning of AGR-1 and TC1 and TC2 readings were available only after the first cycle. The TC drift monitoring plots for two TC pairs in Capsule 4 shown in Figure 12 (pair of TC1 and TC 3 on the top row and pair of TC2 and 1 on the bottom row) reveal that TC1 and TC3 were stable and TC2 drifted down by more than $100^{\circ} \mathrm{C}$ in relation to its simulation.

\subsubsection{TC1 and TC3}

TC1 and TC3 are stable. The TC drift monitoring plots for the TC1-3 pair presented in the Figure 12 (top row) provide adequate evidences that $\mathrm{TC} 1$ and $\mathrm{TC} 3$ responded similarly relative to each other and to their simulation:

- Control charts of TC pair temperature differences (Frame 1): For the TC1-3 pair (top row image), the measured (red dots) TC differences stay within the tight control limit band of $25^{\circ} \mathrm{C}$ parallel to simulated (purple dots) TC differences.

- $\quad$ TC residuals (Frames 2): The residual plots for TC1 (blue dots) and TC3 (green dots) are parallel with each other and clustered around zero, indicating consistency with their simulation. TC3 readings are closer to simulation than TC1 during the first half of AGR-1, then TC1 readings are closer to simulation during the second half of AGR-1.

- Correlation coefficients (Frames 3): The high correlation coefficients for this TC pair indicate the consistency of these TC responses to the capsule thermal condition.

- Actual measured and simulated TC plots (Frame 4): The TC measurements are close to simulated temperatures for both $\mathrm{TC} 1$ and $\mathrm{TC} 3$.

\subsubsection{TC2}

TC2 (center TC) drifted down for about $100^{\circ} \mathrm{C}$ during the first three cycles. The TC drift monitoring plots for the TC2-1 pair as presented in Figure 12 (bottom row) are as follows:

- Control charts of TC pair temperature differences (Frame 1): Because of the different thermal condition in TC1 (peripheral) and TC2 (center) locations, their temperature differences are increasing over irradiation period as shown by simulated TC differences (purple dots). Therefore, the measured TC differences for this TC pair (red dots), while they do not have out-of-control instances during the monitoring period, are much lower than simulated TC differences, indicating that the at least one TC may have drifted relative to simulation. This evidence alone is not sufficient to conclude which TC drifted.

- TC residuals (Frame 2): The TC2 residuals (blue dots) clearly indicate a downward drift during Cycles 139A and 139B for $\sim 100^{\circ} \mathrm{C}$ and stayed at that level over the rest of the AGR-1.

- Correlation coefficients (Frame 3): The correlation coefficients for the TC2-1 pair are scattering lower than 1, indicating that the thermal properties were changing differently for these TC locations.

- Actual measured and simulated TC plots (Frame 4): the TC2 measurements (solid blue line) are dropping lower than the TC2 simulated temperatures (dash blue line) in Cycle 139A, but stay parallel to the simulation after the end of Cycle 139B. 

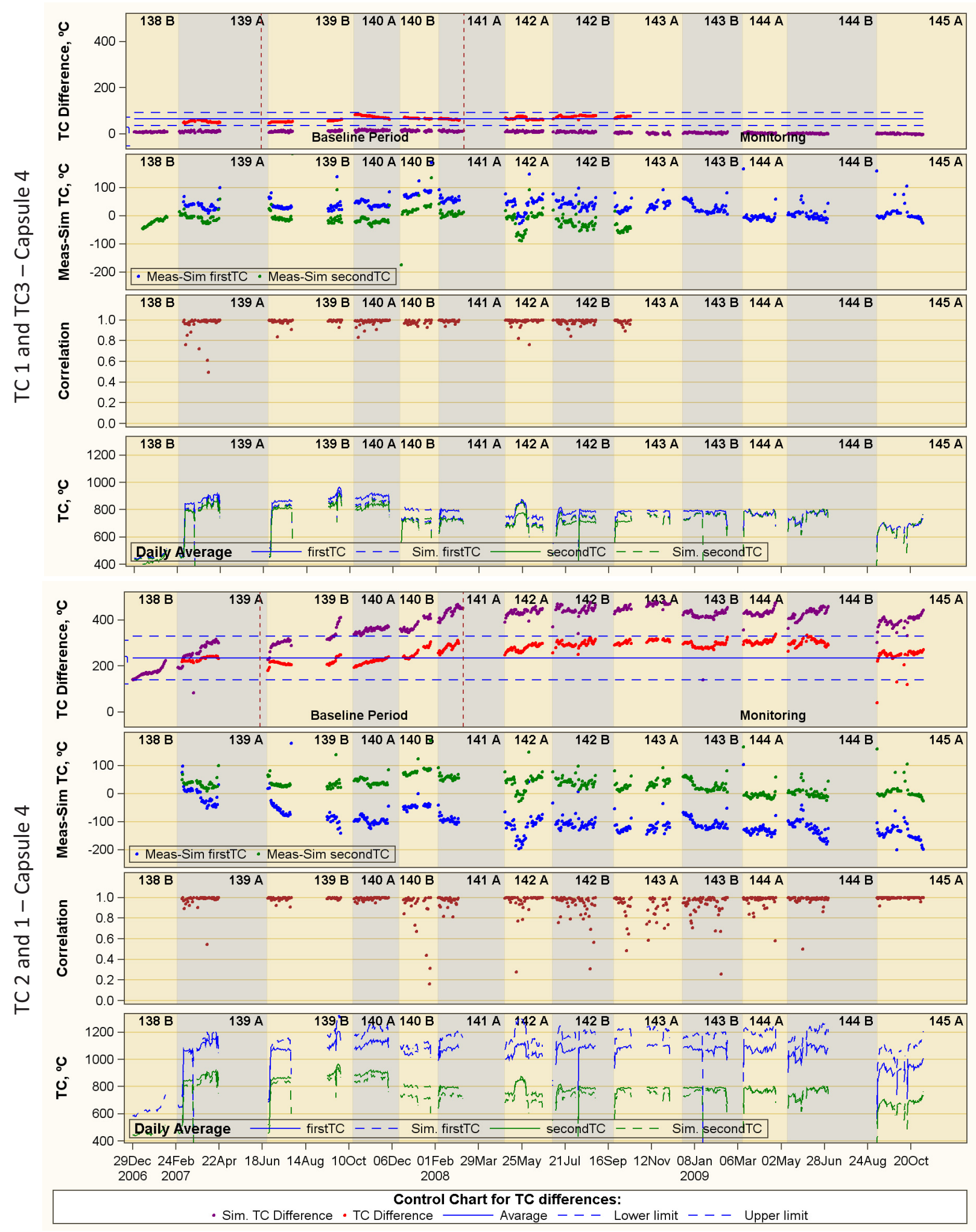

Figure 12. The drift monitoring for TC2 \& 1 and TC1 \& 3 pairs of Capsule 4. 


\subsubsection{Capsule 3 (TC1 is biased up before failure date, TC2 drifted and failed during the second cycle, and TC3 is more or less stable in relation to simulation before failure during Cycle 140A)}

Capsule 3 has three thin wire TCs 1/16 in. in diameter. TC1 and TC2 are molybdenum-niobium type and TC3 is Type-N. Both are located in the capsule periphery. TC1 and TC3 are located in similar locations in the eastern side, shielded from ATR core and inserted at 3 in. and 1 in. depth respectively in the graphite sample holder. TC2 is located in the western periphery facing the ATR core and inserted right at the capsule center level at a 2 in. depth. All three TCs were failed by the program experts, resulting in much less data than from the operational TCs in Capsules 6, 5, and 4. TC1 had data for five cycles, TC2 for 2 weeks during the second cycle, and TC3 for four cycles.

The drift monitoring plots for the TC1-2 pair (top row of Figure 13) was not useful because the TC2 readings were too few (green dots in Frame 2).

The TC drift monitoring plot for TC1-3 pair is presented on the bottom row of Figure 13. TC1 residuals (blue dots in Frame 2) are parallel to the horizontal axis at $100^{\circ} \mathrm{C}$, indicating clear bias in relation to the simulation. TC3 residuals (green dots in Frame 2) vary widely but center around zero, indicating consistency with the simulation before its failure in Cycle 140A.

\subsubsection{Capsule 2}

TC1 was damaged at fabrication, TC2 had qualified readings for only 2 weeks, and TC3 drifted upward for about $100^{\circ} \mathrm{C}$ from the start until its failure date in Cycle 139B.

Capsule 2 has the same three TCs and locations as Capsule 3. All three TCs were failed very early: TC1 was damaged during assembly, TC2 had readings for only a few days in Cycle 139A, and TC3 had readings for first three cycles but failed by the end of Cycle 139B.

Because of the small amount of TC readings available, the drift monitoring plots for the TC2-3 pair (top row of Figure 14) are not helpful. The TC drift detection is based mainly on TC residuals in Frame 2. TC2 residuals (blue dots) were close to $0^{\circ} \mathrm{C}$ during the only 2 weeks where its readings were qualified, which clearly indicates strong drift failure of TC2. TC2 readings after March 22, 2007, were disqualified because of clear and rapid downward drift relative to TC3 readings as well as simulation.

TC3 drifted from the start of the first cycle, where its residuals (green dots in Frame 2) immediately started to drift upward for about $100^{\circ} \mathrm{C}$ until it failed at the end of Cycle 139B.

\subsubsection{Capsule 1}

TC1 was damaged at fabrication; TC2 was stable and failed in Cycle 139B.

Capsule 1 has two thin wire TCs 1/16-in. in diameter. TC1 is molybdenum-niobium type and TC2 is Type-N. They are both located in the capsule eastern periphery shielded from the ATR core and inserted at a 1 in. depth. Therefore TC1 and TC2 should also experience the same temperature level. TC1 was damaged during assembly and TC2 had data for the first three cycles.

Since Capsule 1 has only one TC with some qualified data, there is no meaningful drift monitoring for the TC pair. Therefore, the plots on the bottom row of Figure 14 have only measured and simulated data for TC2. The relatively flat residual plot (Blue dots in Frame 2) shows that TC2 is stable because its readings are consistent with the simulation. The plots of actual measured and simulated TC2 (Frame 4) followed each other closely, pointing to the consistency of TC2 readings in relation to its simulation. 

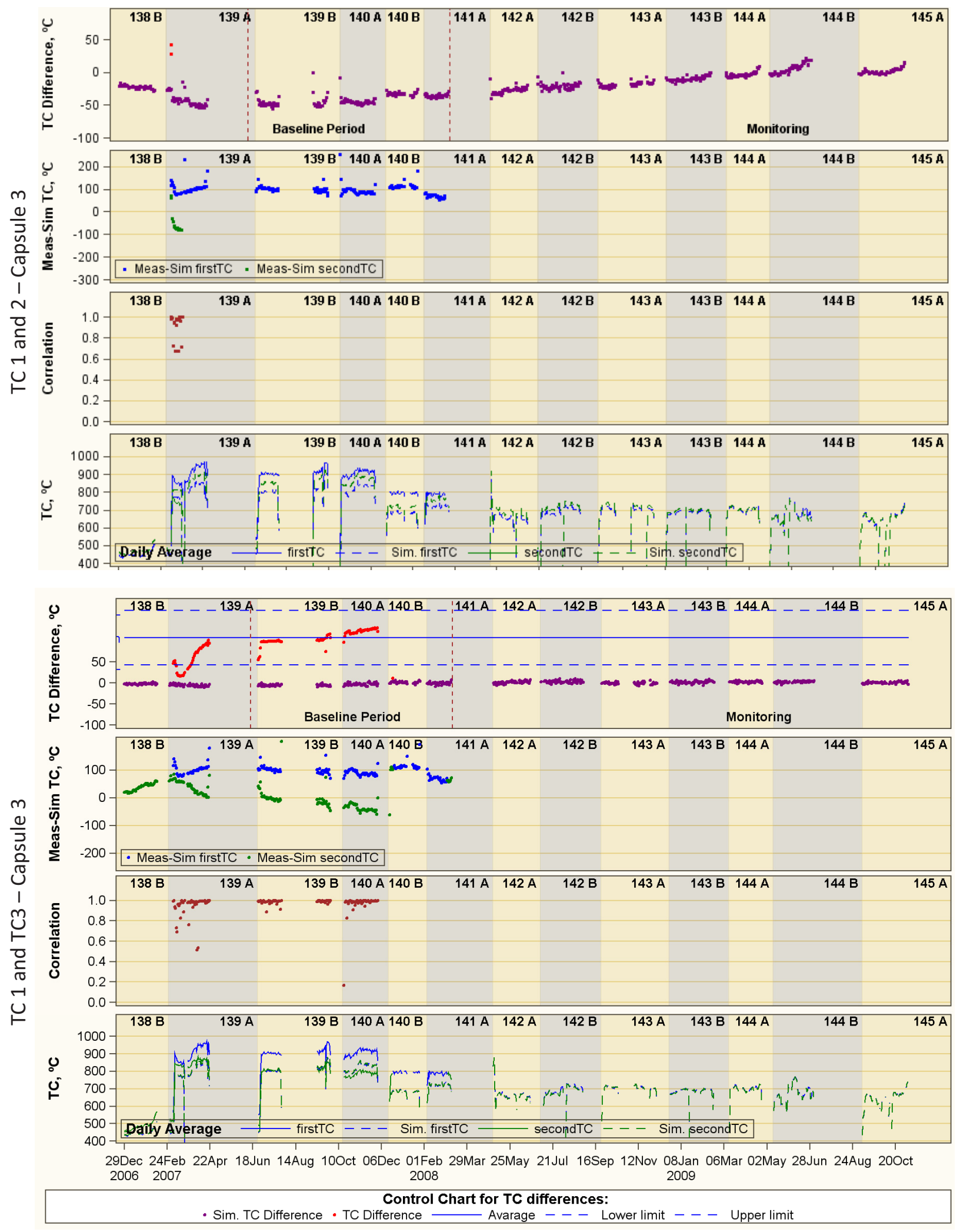

Figure 13. The drift monitoring for TC1 \& 2 and TC1 \& 3 pairs of Capsule 3. 

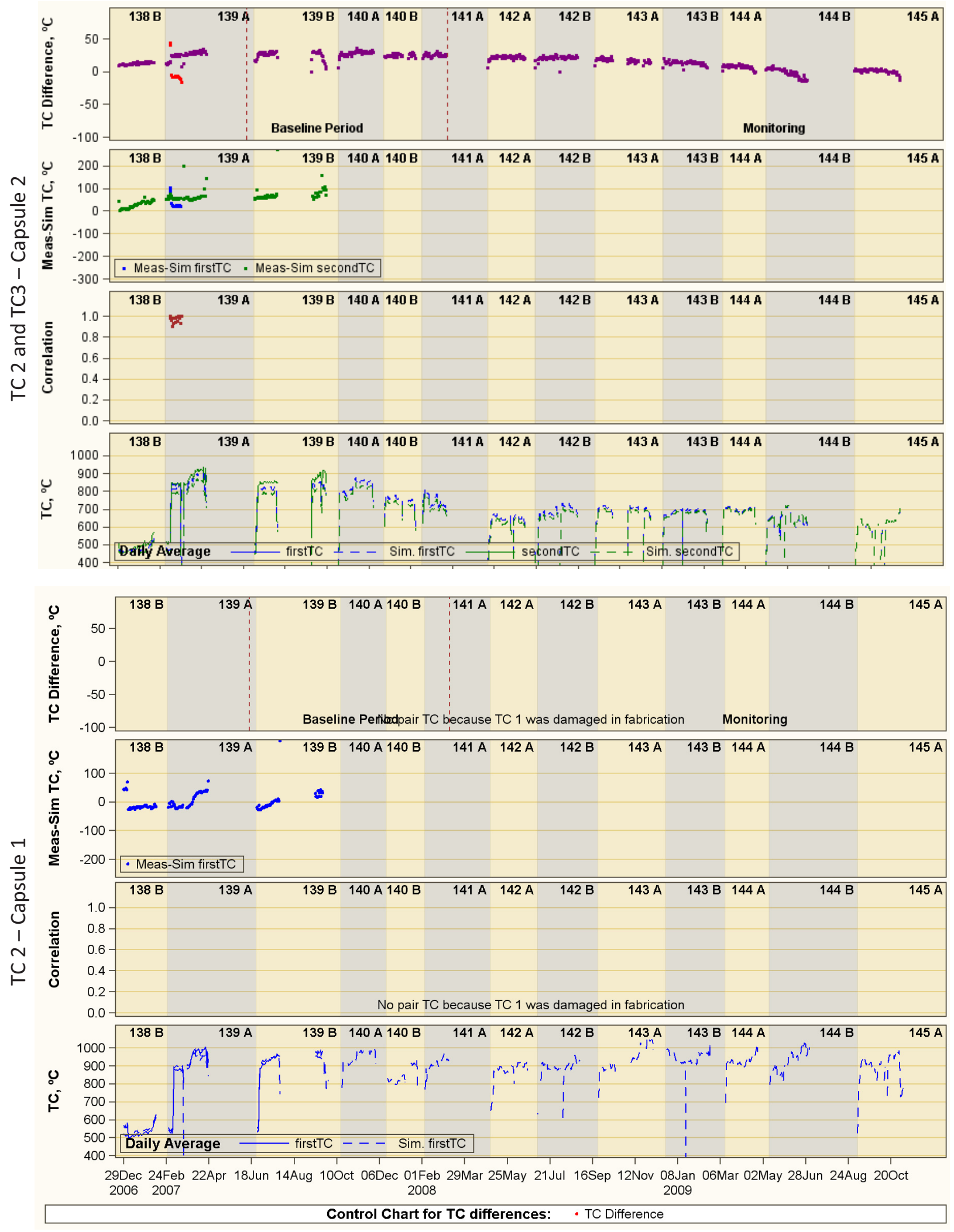

Figure 14. The drift monitoring for TC2 \& 3 pair of capsule 2 and TC2 of Capsule 1. 


\subsection{AGR-1 TC Characteristic and Performance Summary}

Figure 15 summarizes important characteristic and performance information for all TCs in the AGR-1 experiment. The horizontal axis presents the TC identification (capsule\#_TC\#) as follows:

- TC positions in the capsule are shown on top of the green column. The location (such as capsule center, Front north (N), etc.) and insertion depth in the graphite sample holder. The figure on the bottom details the TC position in the capsule. The TC wire diameters $(3 / 32 \mathrm{in}$. for thicker diameter wire and nothing for the 1/16-in wire) are also presented here.

- TC types (molybdenum-niobium or Type-N) are shown under the horizontal axis.

- Identified TC performance status (stable, bias with biased amount, drifts with estimated drifting amount, or fails) are given on the bottom below the TC types.

- TC life span measured in EFPDs from the test beginning to the TC failure date is presented by the blue bars.

- The measured (green bar) and simulated (purple bar) temperatures are averaged over entire AGR1 when ATR is full power (average of the NE, SE and C lobe powers is greater than $20 \mathrm{MW}$ ) and both measured and simulated temperatures exist. The time averages and standard deviations of $\mathrm{TC}$ residuals (red bar and error bar) show the TC bias relative to their simulation.
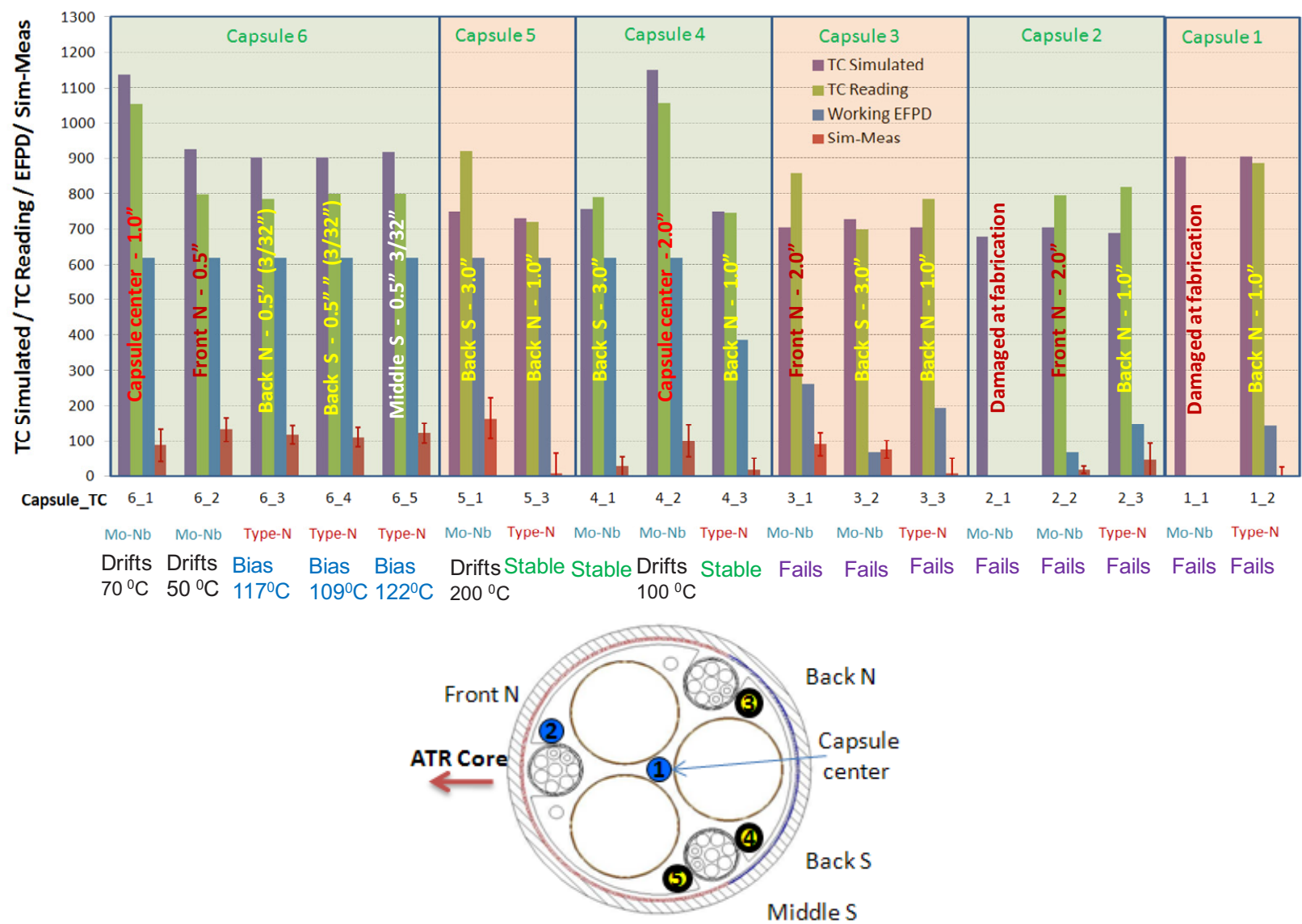

Figure 15. TC characteristic and performance summary for AGR-1 experiment. 


\subsubsection{Main Findings of AGR-1 TC Performance from Data Analysis}

Main findings of AGR-1 TC performance from data analysis are as follows:

- TCs lasted longer (higher blue bars in Figure 15) when they were located in higher capsules in the test train (with Capsule 6 on the top and Capsule 1 on the bottom).

- The simulated temperatures at $\mathrm{TC}$ locations are about $100^{\circ} \mathrm{C}$ higher than $\mathrm{TC}$ readings for all $\mathrm{TCs}$ in Capsule 6, including two Type-N and three molybdenum-niobium type TCs (red bars in Figure 18).

- Among nine surviving TCs:

- TC3 in Capsule 5 and TC1 in Capsule 4 had the best agreement with its simulation according to their drift monitoring plots in Figures 10 and 12. This is also apparent by the low red bar in Figure 15. Thus, these TC readings are valuable in assessing both physics and thermal models. This fact also increases the confidence in the predictability of capsule thermal models, when they are appropriately calibrated to experiment conditions.

- All three TCs with thicker diameter wire (TC3-4-5 in Capsule 6) survived the whole experiment and gave stable readings relative to the simulation with almost the same bias amount of 109 to $122^{\circ} \mathrm{C}$. Since all of them are located in Capsule 6 where the other two thin wire diameter TCs also survived, no comparison can be made to differentiate whether their life span in the test train was because of their wire diameter thickness or because of the top location of Capsule 6 .

However, the fact that all thick Type-N TCs survived and gave stable readings substantiated the decision to exclusively use thick Type-N TCs in the AGR-2 test.

- All four drifted TCs are molybdenum-niobium type. The larger errors on the TC residual red bars in Figure 15 also indicate the possible drift of TC readings, because the increase in the TC residual errors were caused by the residual upward (or downward) slope over time when TCs were drifting (e.g., residuals of TC1 of Capsule 5 in Figure 10).

- Two central TC readings (TC1 in Capsule 6 and TC2 in Capsule 4) drifting down by almost the same amount of about $100^{\circ} \mathrm{C}$ over the AGR-1 irradiation may be an indication of the systematic thermal model bias in calculating temperature at the capsule center location.

- Among seven failed TCs:

- The readings of three type-N TCs (TC3 in Capsule 4, TC3 in Capsule 3, and TC2 in Capsule 1) are consistent with simulation.

- There is no apparent superiority of TC types with thin wire diameter (1/16 in.) in terms of their reliability and durability: excluding two TCs damaged at fabrication (both are molybdenumniobium), there were four Type-N TCs and three molybdenum-niobium TCs that failed relatively early during the AGR-1 irradiation campaign.

- Two TCs were failed during fabrication: TC1 in Capsule 2 and TC1 in Capsule 1.

- Type-N TC readings for all 18 TCs are more likely to be consistent with simulation, while molybdenum-niobium TC readings are likely to drift. 


\section{AGR-1 PHYSICS AND THERMAL SIMULATION DATA ANALYSIS}

This chapter demonstrates that the combined analysis of measured and simulated data leads to important insights for interpretation of simulation results. The TC performance assessment in Chapter 2 found that TC3, TC4, and TC5 in Capsule 6, TC3 in Capsule 5, and especially TC1 and TC3 in Capsule 4 are stable relative to their simulation. Since it is the most consistent with its simulation, data from TC1 and TC3 in Capsule 4 will be used to evaluate both physics and thermal simulation data in accordance with the following steps:

1. Look for behavior differences between measured and simulated TC temperatures based on actual data comparison (e.g., TC residuals) and relationship comparison (e.g., regression functions).

2. Use "zoom-in" technique to magnify the observed trend and minimize number of influencing factors for possible explanation.

3. Attempt to review physics and thermal model assumptions to reveal causes of the observed data trends suggesting model improvement. These observed trends may also help in model systematic uncertainty quantification.

The thermal sensitivity analyses of the ABAQUS model for the AGR-1 capsules have been completed, indicating that the three most important parameters are control gap size, fuel heat rate, and gas composition (Hawkes 2011). Of less importance are the conductivities of the fuel compact and graphite holder and the emissivity of the metallic components. The PIE metrology data of AGR-1 capsule components indicated that all fuel compacts unevenly shrank and the graphite fuel holders unevenly swelled (because of higher boron addition to the graphite) for Capsules 2, 3, 4, and 5 (the middle capsules), but shrank for Capsules 1 and 6 (with less boron concentration). As the result, the control gap shrank for four middle capsules, while the gap between the graphite body and the fuel compact increased, offsetting somewhat the reduction in control gap. This behavior is being taken into account when calculating the AGR-1 temperatures. The control gap changes complicate simulation data analysis because of the additional thermal model assumption that the gap distance is evenly and linearly reducing for the middle four capsules and slightly increasing for top and bottom capsules over the entire AGR-1 irradiation from the initial actual hot gap size (as-fabricated gap size minus thermal expansion of graphite holder) to the estimated end gap. Because there was no fuel particle failure in AGR-1, the R/B from the heavy metal contamination is very sensitive to temperature, and thus is a measure of the temperature in each capsule. The end gap sizes were therefore optimized, maximizing correlation between the temporal temperature predictions and the temporal $\mathrm{R} / \mathrm{B}$, indicating that the thermal model is capturing all of the important physical phenomena. The reduction of the end gap for four middle capsules could be as much as $50 \%$ of initial gap.

Figure 16 shows the TC residuals (measured minus simulated temperatures at TC location) for all AGR-1 operating TCs in six capsules as a function of the EFPD and ATR cycles. The clear forms of TC residual trend are typical indications of simulation bias. According to this figure, the following TC residual trends are observed for:

1. Cycle $138 B$ - The upward TC residual trend for about $50^{\circ} \mathrm{C}$ is clearly apparent for TC 3 in all four capsules located in the middle of AGR-1 test train (Capsule 2-3-4-5), but at the same time TC residual trend is flat for TC1 in Capsule 1 (bottom capsule) and TC3 in Capsule 6 (top capsule).

2. Cycle $139 \mathrm{~A}$ - The TC measurements and simulation results are closest to each other for all stable TCs (their TC residuals are nearly flat). This may be explained by the fact that this cycle data were used in the ABAQUS thermal model calibration, thus any initial bias in model inputs were compensated obscuring TC residual trend. 
3. Remaining 11 cycles - The upward trend of TC residuals within each ATR cycle was most apparent for all TCs in Capsule 6 during all 11 cycles and for TC1 in Capsule 1 during the third cycle - the last cycle that this TC had valid readings. However, this trend was not present for other stable TCs in Capsules 2, 3, 4, and 5 located in the middle of the test train.

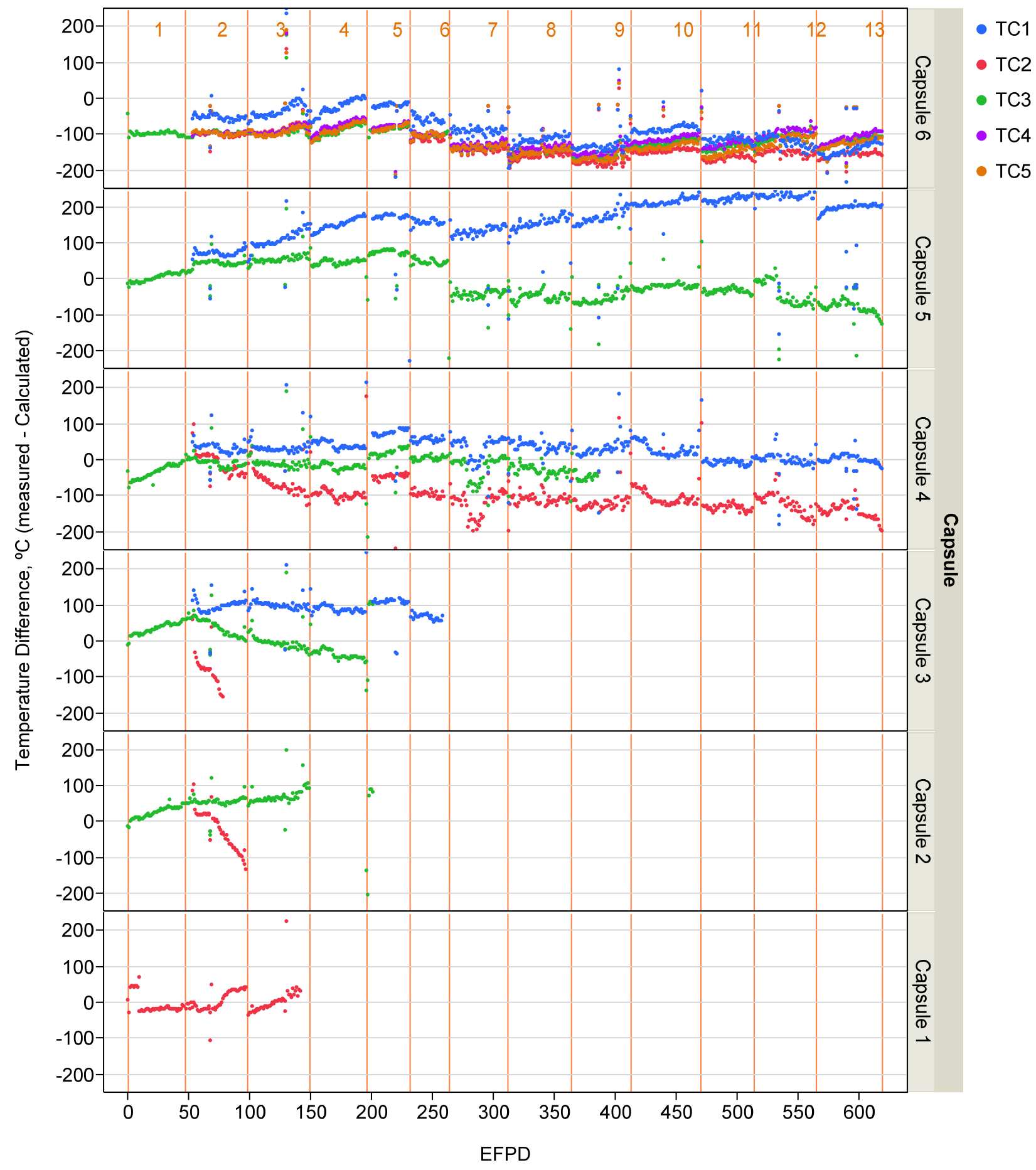

Figure 16. Difference between measured and predicted TC temperatures. 


\subsection{Underestimation of Fission Power at the End of an ATR Cycle}

\subsubsection{Upward TC Residual Trends for TC3 in Four Middle Capsules During the First Cycle}

During the first cycle, the AGR-1 capsules were run on cold temperatures with pure helium for both capsule flow and leadout flow leading to unchanged capsule gas compositions. Over this early time in the AGR-1 experiment, it is reasonable to assume that the changes of control gap for the middle capsules could be negligible. Therefore the TC residual upward trend for TC3 in the middle capsules can only be explained by the increasingly higher heat rates of the actual components (such as fuel and graphite) in these capsules over the first cycle compared to the simulated value. In other words, capsule fission powers were underestimated at the end-of-cycle.

The difference in the control gap behavior between the four middle capsules and the two edge capsules mentioned above might be one of the factors contributing to differences in this TC residual trend. The other factor may be the unevenness of the ATR core spatial neutron flux profile near the top (Capsule 6) and bottom (Capsule 1) capsules of the AGR-1 test train.

\subsubsection{TC Residual Upward Trend within Each ATR Cycle for TCs in Capsule 6}

As stated previously, the control gap, fuel fission power, graphite heat rate, and control helium-neon gas mixture (neon fraction) are primary factors determining capsule temperature. The zoom-in technique is used to demonstrate the observed trend and minimize the number of influencing factors for possible explanation. Figure 17 shows measured and simulated TC1 and TC3 in Capsule 6 together with relevant parameters' data zoomed-in to the time span from 150 to 200 EFPDs (Cycle 140A). This time span was selected because the neon fraction (Frame 3) and the fuel and graphite heat rates (brown and black dots in Frame 4) are relatively unchanged. The unchanged capsule fission powers and neon fraction (unchanged gas mixture thermal conductivity) lead to relatively constant simulated TC temperatures (dashed lines in Frame 1). However, during the same period, the TC readings (solid lines in Frame 1) were increasing. These behavior differences between measured and simulated TCs over this selected time span are more apparent by the TC residual upward trend in Frame 2 for both TC1 and TC3. The increase in capsule temperature felt by TCs within one ATR cycle was confirmed by the increase of fission product R/Bs of radionuclides $\mathrm{Kr}-85 \mathrm{M}, \mathrm{Kr}-88$, and $\mathrm{Xe}-135$ over that time (Frame 5). These increases point to possibly higher capsule temperatures by the end of a cycle than predicted by the thermal model.

It might be reasonable to assume that the changes in thermal properties of the capsule components (e.g., change in gas gap because of fast fluence induced fuel compact shrinkage and heat induced graphite

holder expansion) could be negligible within a short time period. Since the neon fraction measurement is not time dependent, the increase in actual capsule temperature indicates that the actual capsule fission powers by the end of each ATR cycle (both fuel compacts and graphite holder) is higher than their prediction by the JMOCUP depletion model. In other words, this model might be more accurate for capsule powers at the beginning than at the end-of-cycle. Thus, the JMOCUP depletion model assumptions and limitations were carefully studied looking for plausible reasons for underestimation of capsule power toward the end-of-cycle for Capsule 6.

The fact that the end-gap sizes were optimized, maximizing correlation between the temporal temperature predictions and the temporal R/B for four middle capsules may compensate for this fission power underestimation within each cycle in the four middle capsules. Thus, this TC residual upward trend was obscured for these capsules. 


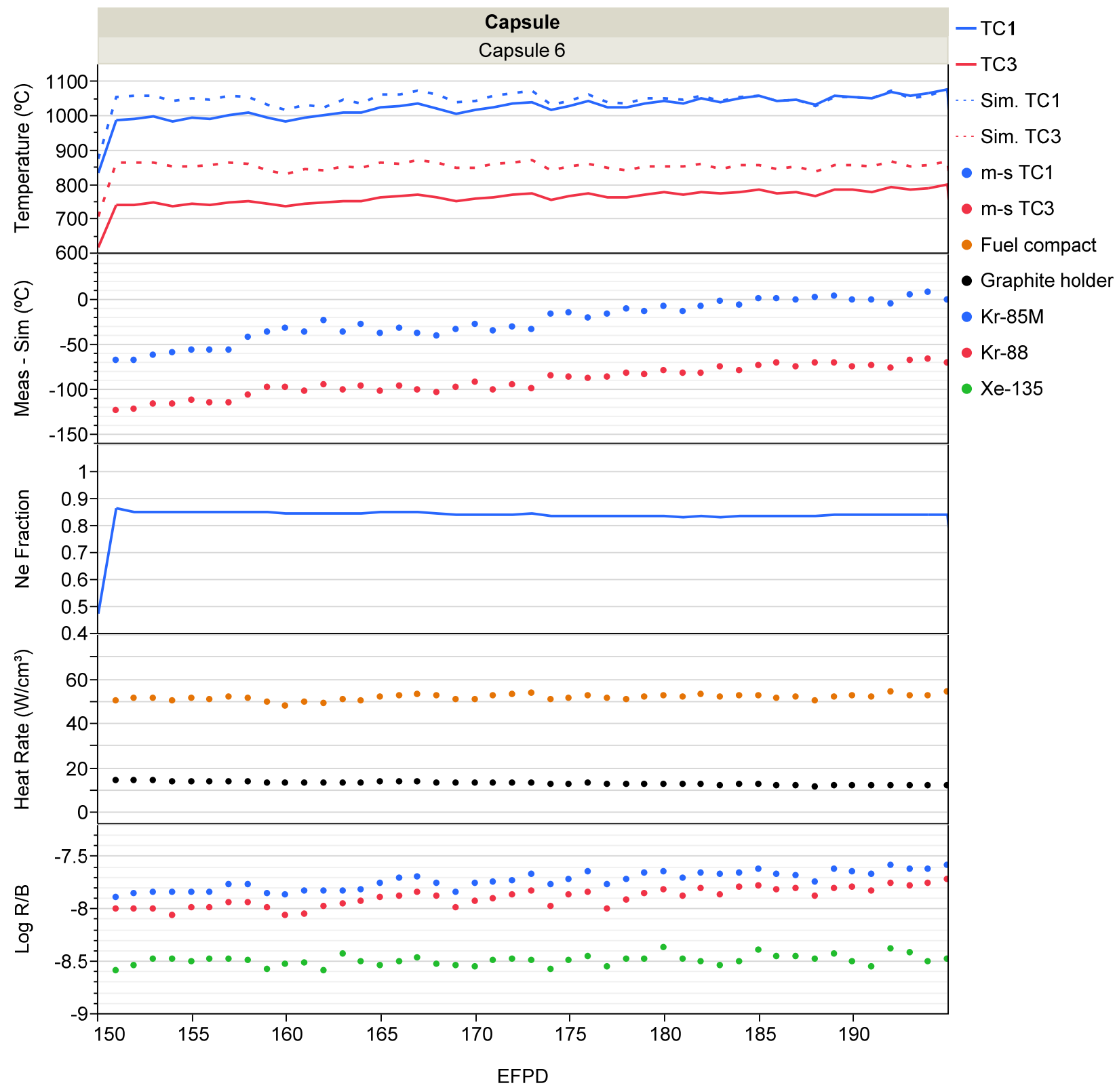

Figure 17. TC1 and TC3 in Capsule 6 and relevant parameters data from 150 to 200 EFPDs.

\subsubsection{JMOCUP Depletion Model May Underestimate Capsule Power at the End of a Cycle}

According to the JMOCUP model ECAR (Sterbentz 2011), the following possibilities for JMOCUP depletion analysis underestimation of fuel and graphite power densities toward the end of each ATR cycle in relation to the cycle beginning were found:

- The calculated k-effective expected to hover around 1.00 (or critical) became more positive over the later third of the cycle. This k-effective increase above 1.00 toward the end of the cycle will affect the calculated AGR-1 fuel power distribution within one cycle. 
- The calculated ATR fuel was depleted more at the beginning than at the end-of-cycle. This may mean that the calculated neutron flux reduced at the same level of total core power at the cycle end and caused reduction in AGR-1 fuel fission power.

- Finally, the comparison of calculated versus lobe powers shows that the combined $\mathrm{NE}+\mathrm{C}+\mathrm{SE}$ lobe powers are $3.23 \%$ higher than the measured combined value at Day 8 and reduced to $1.26 \%$ at the end-of-cycle. This means that the starting source neutrons in the MCNP calculation are favored slightly for these three lobes and would probably result in a slight increase in the fastneutron flux track tally in the B-10 AGR-1 test facility and, correspondingly, as well as a slightly higher thermal-neutron flux track tally, but probably to a smaller degree. This explains the reduced AGR-1fuel fission power toward the end of a cycle.

\subsection{Overestimation of TC Temperature with Increasing Neon Fraction}

This section presents evidence of the ABAQUS thermal model over predicting temperature relative to $\mathrm{TC}$ readings with increase of neon fraction. Three comparative methods were applied for readings from TC1-3 in Capsule 5, and TC 1-3 in Capsule 4 to demonstrate the thermal simulation trend of (1) zoom-in plots, (2) JMP multivariate platform, and (3) regression analysis.

\subsubsection{Evidence Based on Zoom-in of Irradiation Parameters Plots}

In order to reveal this simulation trend, time spans, when the neon fraction jumps from a lower level to a significantly higher level while the fuel fission powers stayed relatively constant, were sought. As a result, the period spanning from 240 to 290 EFPD was selected for zoom-in analysis for Capsule 5 as the example in Figure 18 shows, and Capsule 4 as the example in Figure 19 shows. According to Figure 18, at the middle of this period, the neon fraction in Capsule 5 jumped from nearly 0 to 0.8 (Frame 3), while fuel and graphite power densities (green and brown lines in Frame 4) are largely unchanged. In response to the big jump in neon fraction, both measured and simulated TC temperatures (blue and red lines in Frame 1) increased as well as the fission product R/B (Frame 5), but not by the same amount. The step down in TC residuals (Frame 2) after the neon fraction jump showed that the TC simulated temperature increases more than TC readings in response to significant neon fraction increase. This difference in measured and simulated data of both TCs in Capsule 5 indicates that the thermal model may over predict temperature relative to $\mathrm{TC}$ readings when the neon fraction increases. The same differences between measured and simulated TC values with neon fraction increase were also observed in Capsule 4 as shown in Figure 19. The consistency of this trend across capsules indicates that the error is global, not capsule specific. 


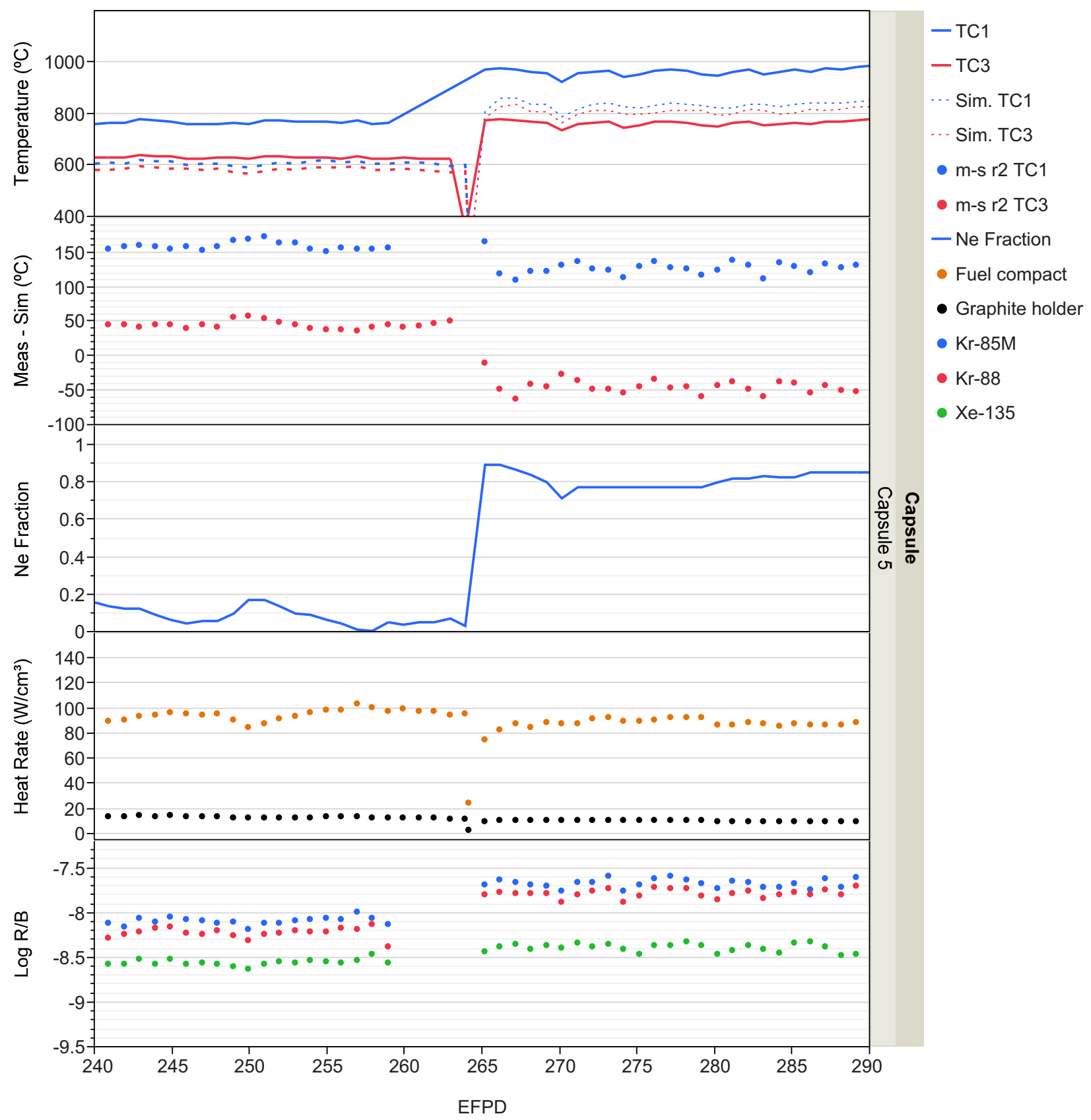

Figure 18. TC1 and TC3 in Capsule 5 and relevant parameters data from 240 to 300 EFPDs. 


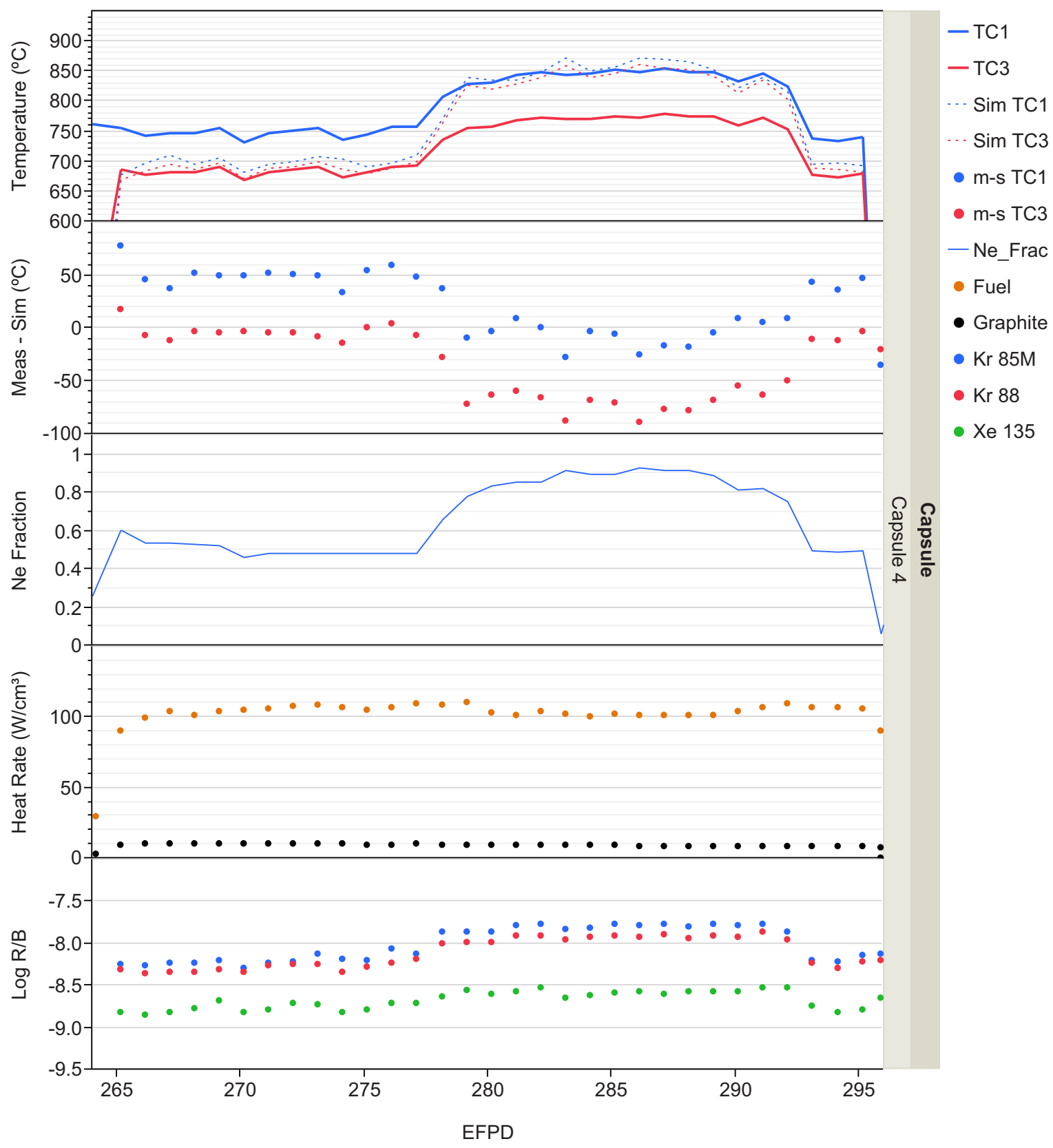

Figure 19. TC1 and TC3 in Capsule 4 and relevant parameters data from 265 to 295 EFPDs.

\subsubsection{Evidence Based on Regression Analysis}

Regression analysis refers to statistical techniques for the modeling and analysis of numerical data consisting of values of a dependent variable (e.g., TC temperature) and of one or more independent variables or predictors (e.g., fission power and neon fraction). The response surface function for temperature $(\mathrm{T})$ is expressed as

$T=\beta_{0}+\beta_{1} t_{E F P D}+\beta_{2} q_{F}+\beta_{3} q_{F}^{2}+\beta_{4} f_{N e}+\beta_{5} f_{N e}^{2}+\beta_{6} q_{F} * f_{N e}$

where $t_{E F P D}$ is effective full-power in days, $q_{F}$ is fuel fission power density $\left(\mathrm{W} / \mathrm{cm}^{3}\right)$, and $f_{N e}$ is neon fraction. 
This regression analysis is used to study the relationships of TC measurements and simulations as a function of calculated fuel power densities, neon fraction, and EFPD to find differences in simulation behavior in response to thermal condition changes. The regression models (Eq. 1) fit very well to measured and simulated temperatures for both TCs with slightly better fit for the simulated temperature (high determination coefficient R2 $=0.93$ for measured TC1 and 0.99 for simulated TC1, and small root mean square errors $=23.8^{\circ} \mathrm{C}$ for measured $\mathrm{TC} 1$ and $11.9^{\circ} \mathrm{C}$ for simulated $\mathrm{TC} 1$ ). Therefore, these fitting models can be used for evaluation of the relationship differences between measured and simulated TC temperatures as regression functions of thermal condition parameters.

The prediction profilers in the JMP "fit model" platform displaying profile traces for each independent variable are shown in Figure 20 for both Capsule 5 measured and simulated TC 3 values together with averaged fuel temperature. A profile trace is the predicted response as one variable is changed while the others are held constant at the current values (SAS 2009). The squared terms of the regression model (Eq. 1) for the fuel power density and neon fraction lead to the curved traces (Columns 2 and 3). The curvature differences of the profiles between measured (top panel) and simulated (middle panel) TC3 values for neon fraction vary - the simulated TC3 values are more sensitive to neon fraction than actual TC readings (higher sensitivity triangle and steeper slope) and, more importantly, the sign of the neon fraction second-order term is positive for simulated TC1 (concaved curvature) and slightly negative for measured TC1 (convex curvature). At the same time, the neon fraction curvature is the same for simulated TC3 and averaged fuel temperature (bottom panel). This evidence leads to the conclusion that the predicted temperatures by ABAQUS thermal model increase more than TC readings when neon fraction increases.

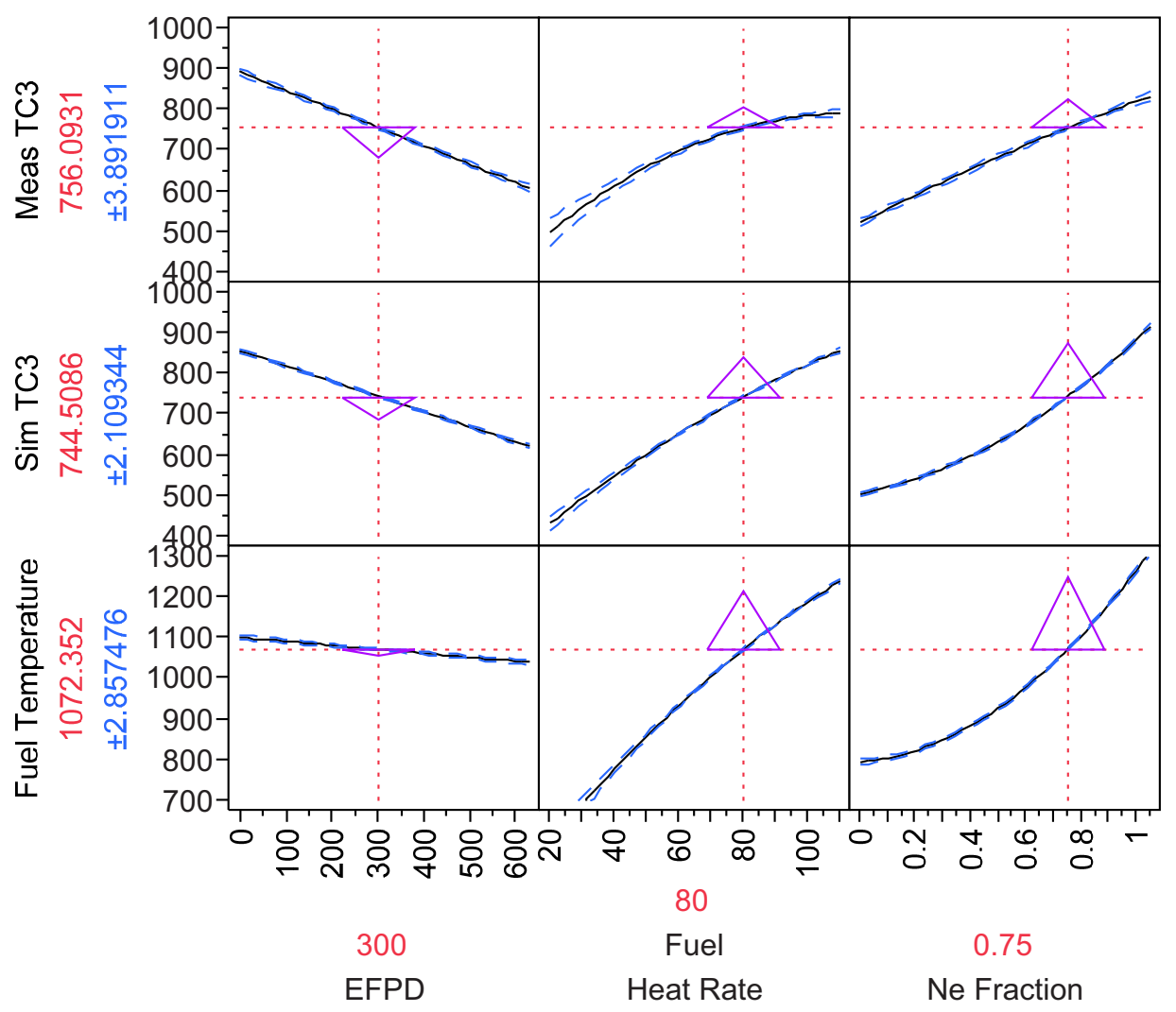

Figure 20. Prediction profilers of Capsule 5 measured and simulated TC3 values and fuel temperatures. 


\section{STATISTICS-BASED SIMULATION-AIDED EXPERIMENT CONTROL}

\subsection{AGR Temperature Control}

As noted early in this report, the AGR-1 test involved several capsules, each comprised of fuel compacts under testing. The thermal conditions in the test section are monitored by TCs whose readings are exhibited on the Distributed Control System (INL 2009c). The target fuel temperatures are defined in the AGR test specification (Maki 2004). The fuel compact temperatures are important both for characterization of the test conditions needed for validation of future fuel performance simulation codes and for qualification of fuel performance in support of VHTR licensing. Success of the experiment campaign depends on whether the target fuel temperatures are stably maintained during the entire irradiation period. During the test operation, a gas gap between each capsule and the contained specimens receives a continuous supply of blended gases (helium and neon) that have vastly different thermal conductivities. As a minimum, gas blenders shall be capable of providing control blends in the range of $100 \%$ helium to $100 \%$ neon. Using readings from the control TCs in the test train, the gas mixture is adjusted to maintain the TC temperature in that capsule within $5^{\circ} \mathrm{C}\left(10^{\circ} \mathrm{F}\right)$ of the indicated temperature with reactor power at steady-state $( \pm 2 \%)$. To this effect, the gas supply system must be able to adjust the gas mixture content at a frequency greater than the transportation time to the capsule (INL 2009c). The blending operations are performed in a mass flow controller cabinet linked to a computer control console in the loop operation control system area. The irradiation test vehicle gas control system monitors the gas supply manifold lineup and status. Each new bottle added to the supply manifold is automatically tested for moisture and gas composition to ensure the correct gas has been inserted in the correct position. All gas bottles, except for helium-3 (used in abnormal case of high power in ATR core - known as PALM), are tested and placed online and offline automatically, and flow to each capsule shall be controlled such that total flow (helium plus neon) is less than $50 \mathrm{sccm}$.

The input required for the capsule temperature control includes capsule TC temperatures, control mode (manual, automatic, purge), and selection of the control TC input signal. The latter provides the process feedback to the control configuration. The output from the temperature control configuration is the control signal for the helium and neon mass flow controllers.

Since the TC temperature is essential for capsule temperature control, it is important that TC readings correctly represent the capsule thermal condition. However, because of harsh operating conditions in the irradiation environment, a significant fraction of TCs in capsules may deteriorate and fail during the long duration of fuel testing. The three statistical methods discussed in Section 2 help identify early indication of TC malfunction such as drift or virtual junction. Furthermore, the capability of the statistical tools can be augmented by making use of numerical data from physics-based simulations that are run on a daily basis and stored in NDMAS (Abbott 2010). These data include the fuel temperatures, which also are used as the test target quantities.

Most notably, correlation analysis performed for the simulation-estimated fuel temperatures and the TC readings reveals a strong correlation. Since the gas mixture is adjusted only when the reactor power is at steady -state (or at full power), the simulated fuel temperatures and TC readings during that time only are selected for temperature control analysis. Table 2 presents the correlation coefficients between the capsule average and peak fuel temperatures and TCs readings in AGR-1 Capsule 5. As expected, the correlation coefficients are fairly high between the peak fuel temperature and TC readings (from 0.88 for TC1 to 0.80 for TC3) and average fuel temperature (0.9966). This allows the linear regression function (e.g., Eq. 2) to be established based on past data and used to estimate the actual fuel temperature from the $\mathrm{TC}$ readings or vice versa. 
Table 1. Correlation coefficients between fuel temperatures and the TCs readings in Capsule 5.

\begin{tabular}{l|ccc}
\hline & TC_1 & TC_3 & Average Fuel Temperature \\
\hline Peak Fuel Temperature & 0.8855 & 0.8012 & 0.9966 \\
\hline
\end{tabular}

The scatter plots for Capsule 4 measured TC1, simulated TC1, and averaged fuel temperatures (third panel of Figure 21), show that in order to keep fuel temperature at $1100^{\circ} \mathrm{C}$, the $\mathrm{TC} 1$ input signal (first row) should drop from $880^{\circ} \mathrm{C}$ for Cycle $139 \mathrm{~A}$ (red line) to $680^{\circ} \mathrm{C}$ for Cycle $145 \mathrm{~A}$ (dark pink line). The same change in simulated TC1 at given simulated fuel temperature is shown in the second row. These changes in TC and fuel temperature relationship prompts the update of the control TC input signal over time as proposed below.

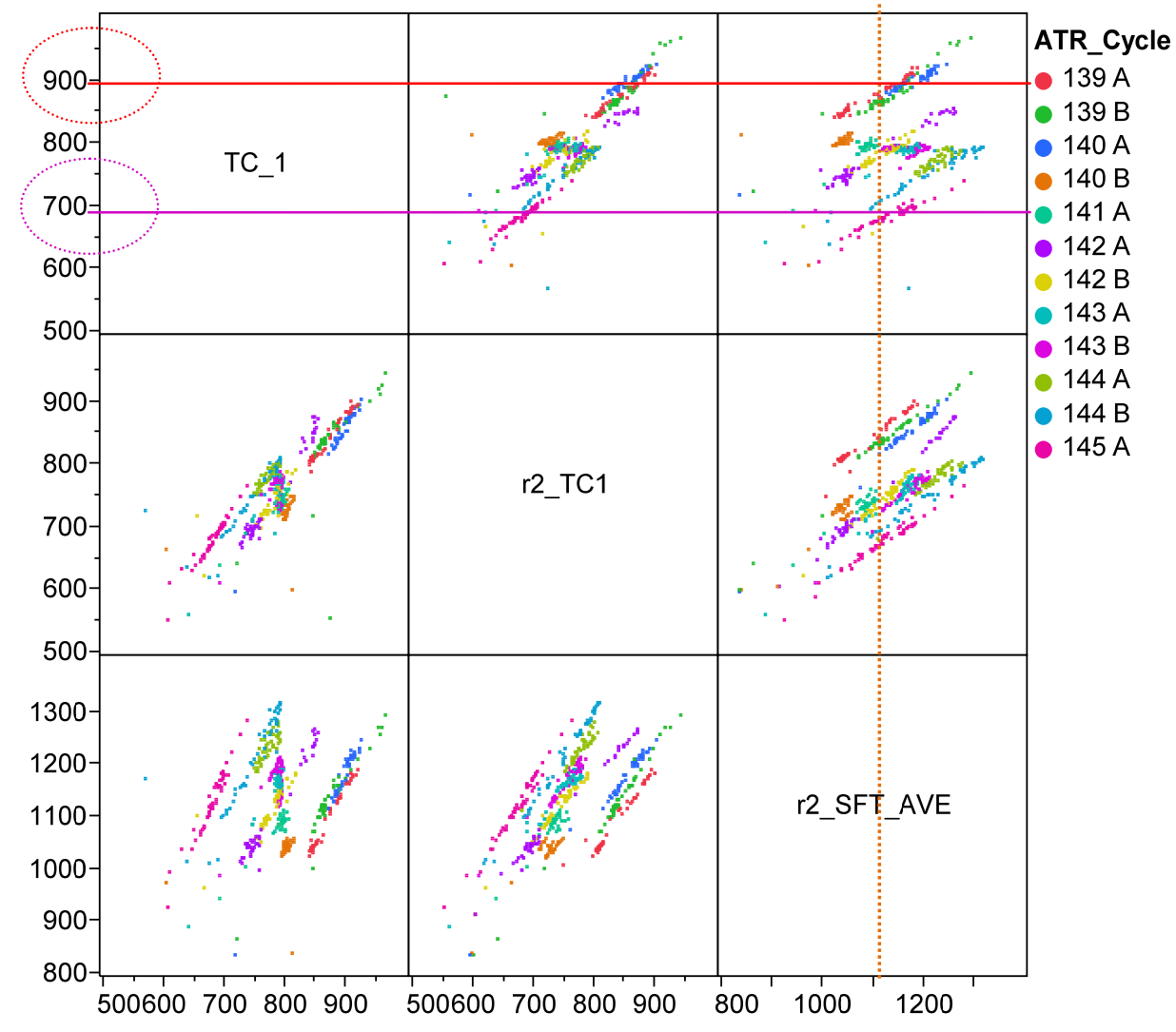

Figure 21. Capsule 4 measured and simulated TC1 values and fuel temperatures for all ATR cycles.

\subsection{Direct regression procedure}

The main motivation for developing a statistics-based simulation-aided experimental control procedure for AGR tests is the recognition that the current control strategy of maintaining a constant value of a control TC fails to recognize significant variability of temperature differences between the fuel target temperature and the control TC-measured value. The variability stems from both changes in AGRexternal physical conditions (neutron flux profile) and AGR-internal materials/geometry during the course of testing, as well as possible deterioration of the TC under a harsh operating environment. The main idea of the proposed control method is to account for the variability by using direct relationship between calculated target fuel temperature and actual TC measurement. 
Figure 22 depicts the simulated (red dots) and the regression-based (blue dots) estimates of the peak fuel temperature in Capsule 6 as linear functions of TC1 constructed for all AGR-1 cycles. Their close fit demonstrates that the target quantity of the peak fuel temperature can be evaluated with high confidence from the control TC readings. This direct relationship between fuel target temperature and TC measurement can also be used to control the fuel temperature by setting the control TC input signal ( $T_{T C}^{C O N T R O L}$ ) to the value corresponding to the specified fuel temperature $\left(T_{F U E L}^{\text {TARGET }}\right.$-either peak fuel temperature or capsule average fuel temperature). The high correlation coefficient between the capsule average and peak fuel temperature $(r=0.9978)$ also suggests that it suffices to use one type of fuel temperature to control, since the other could be estimated from the controlled quantity. The procedure proposed for controlling the AGR experiment is as follows:

1. Construct a regression model (estimate the regression coefficients $a_{E F P D}$ and $b_{E F P D}$ of Equation 2 at particular point in time or EFPD) to directly relate the control TC readings as a function of the calculated fuel temperatures $T_{F U E L}^{C A L C}$ based on available results from daily thermal simulations.

$$
T_{T C}^{C O N T R O L}=a_{E F P D} T_{F U E L}^{C A L C}+b_{E F P D}
$$

2. Substitute the target fuel temperature $T_{F U E L}^{T A R G E T}$ into $T_{F U E L}^{C A L C}$ in Eq. (2) to estimate the "control TC input signal" $T_{T C}^{C O N T R O L}$ needed for control set point in the capsule temperature control system.

3. Periodically update regression coefficients $a_{E F P D}$ and $b_{E F P D}$ of Eq. (2) and subsequently the "control TC input signal" using new simulated fuel temperatures and TC readings as the experiment progresses to count for possible changes in their regression relationship.

Thus, given the assumption about linear heat transfer in the AGR fuel and graphite domain, the experiment control can be achieved by making use of the readings from potential deteriorated TCs without having to explicitly characterize the degree of drifting or a bias in the TC readings.

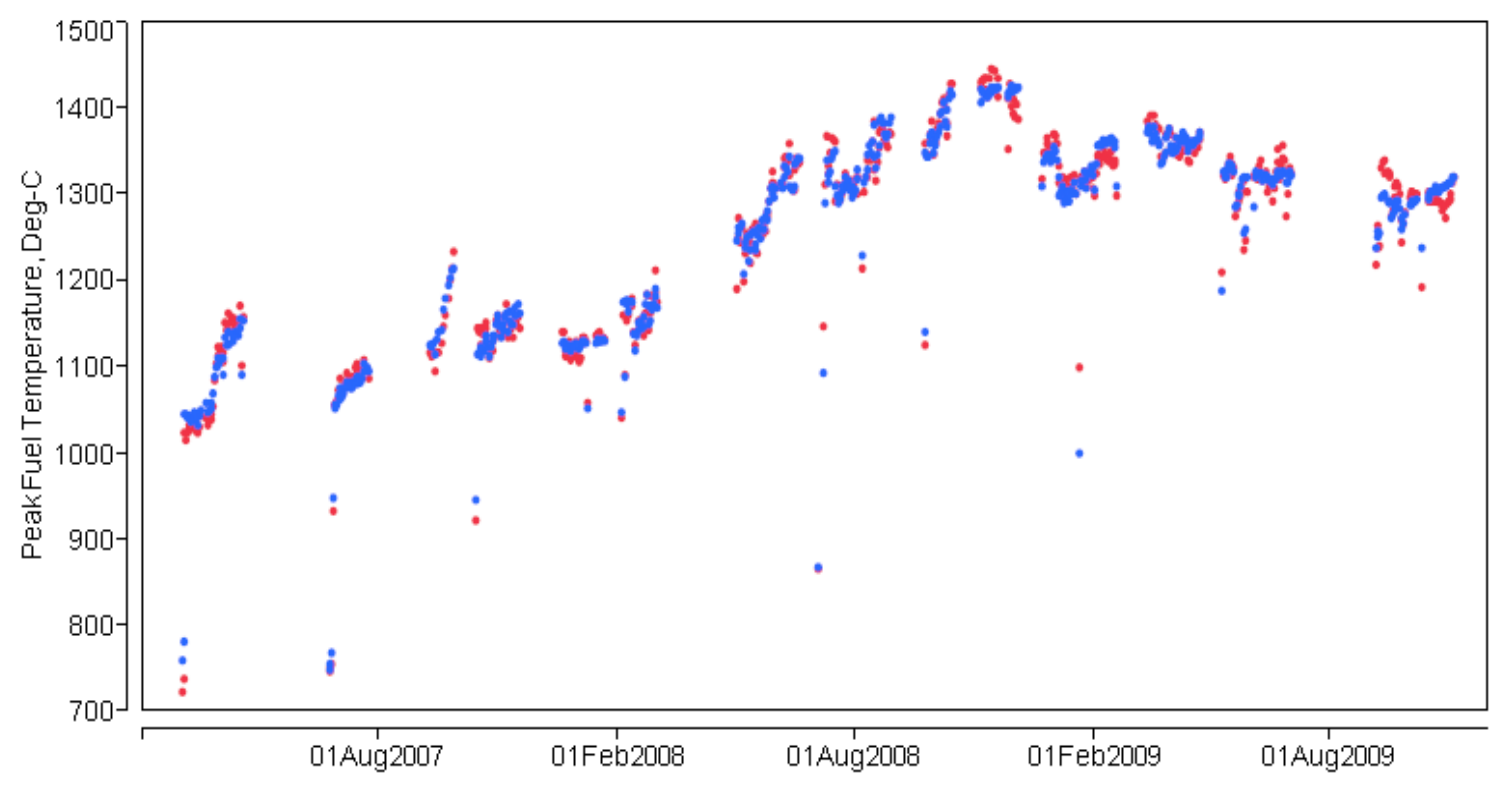

Figure 22. Capsule 6 peak fuel temperatures: regression (blue dots) and simulated (red dots). 


\subsection{Indirect Regression Procedure}

\subsubsection{Proposed Procedure}

Use prediction profilers in JMP "Fit Model" platform applied for both calculated target fuel temperature and actual TC measurement for AGR temperature control.

The prediction profilers of the JMP fit model platform displaying dependent variable profile traces (SAS 2009) for both control TC reading and calculated fuel temperature are presented in Figures 24 and 25. The response prediction profiler not only allows a closer look at the response surface, but also helps find the settings that produce the best response target. For this purpose, the desirability trace was added for each independent variable on the bottom row of the prediction profiler. The profiler also contains a Desirability column, which graphs desirability on a scale from 0 to 1 and has an adjustable desirability function (e.g., match target response) for each dependent variable. The overall desirability measure, defined as the geometric mean of the desirability for each response, is on the bottom of the desirability traces. The regression models for both measured TC and simulated fuel temperature as functions of EFPD, second-order of fuel and graphite power densities $\left(\mathrm{q}_{\mathrm{gr}}\right)$, and neon fraction will be used to maintain the test predefined target fuel temperature during the experiment. The full regression equation for fuel temperature control is expressed as follow:

$T=\beta_{0}+\beta_{1} t_{E F P D}+\beta_{2} q_{F}+\beta_{3} q_{F}^{2}+\beta_{4} f_{N e}+\beta_{5} f_{N e}^{2}+\beta_{6} q_{F} * f_{N e}+\beta_{7} q_{g r}+\beta_{8} q_{g r}^{2}$

The procedure proposed for controlling the AGR experiment based on the JMP prediction profilers is as follows:

1. Construct regression functions similar to Equation 3 together for both measured TC and simulated target fuel temperature (e.g., capsule average fuel temperature) data as they become available using JMP Fit Model platform.

2. Set the desirability function with desired fuel temperature.

3. Select parameters, such as EFPD, fuel fission power, and graphite gamma heat densities, based on the past simulated data, and adjust neon fraction to get desired fuel temperature.

4. Record the corresponding control TC reading to serve as the control TC input signal needed for controlling the set point in the capsule temperature control system for the next time period.

Since the relationship between fuel temperature and TC reading may change over time because of changes in TC and test conditions, the regression functions and subsequently the control TC input signal need to be updated by repeating Steps 1 through 4 using new available measured control TC and simulated fuel temperature data to account for these changes.

\subsubsection{Algorithmic Testing on AGR-1 Examples}

Two examples are analyzed in detail to demonstrate how the proposed procedure for control target fuel temperature facilitates adjustment for changes of the relationship between fuel temperature and control TC reading caused by changes in both TC and test conditions. The first example uses the identified drifted TC1 in Capsule 5 as a control TC (blue dots show a upward drift of TC1 residuals in Figure 23) and the second example used the AGR-1 stable TC3 in Capsule 5 (red dots showing TC3 residuals were around zero) as a control TC to maintain the same target volume-average fuel temperature in capsule 5 of $1100^{\circ} \mathrm{C}$. Each example applies the control procedure for two periods: before 264 EFPD (end of Cycle 141A) named "before" period; and by the end of the experiment named "after" period. Temperature control for Capsule 5 was selected for demonstration because of the big decrease in the control gas gap and increase in the gap between fuel compacts and graphite holder by the end of AGR-1. 


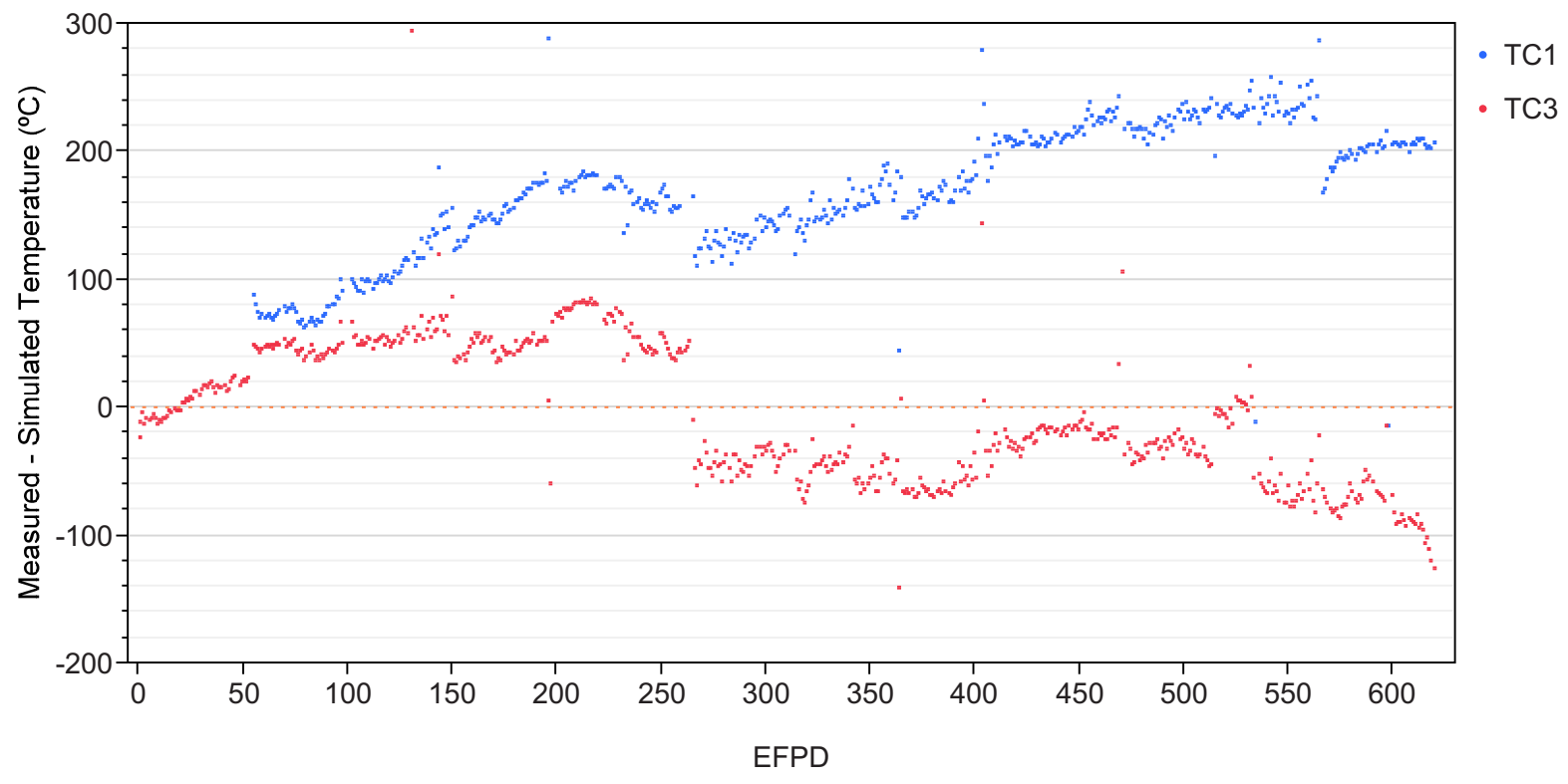

Figure 23. Temperature residuals of TC1 and TC3 in Capsule 5.

\subsubsection{Example 1: Assume TC1 is the Control TC in Capsule 5}

The prediction profilers and desirability functions of fuel power, graphite power, and neon fraction for measured TC1 and simulated fuel temperature using "before" period data are presented on the top row and using "after" period data are in the bottom row in Figure 24. The corresponding "control TC1 input signal" right after 264 EFPD and by the end of AGR-1 is determined as follows:

- For before period (top row), the selected parameters in Step 3 are 100 EFPD, the graphite heat rate was $25 \mathrm{~W} / \mathrm{cm}^{3}$, the fuel heat rate was $65 \mathrm{~W} / \mathrm{cm}^{3}$, and the neon fraction is adjusted to 0.9 for the fuel temperature to reach the desired temperature of $1100^{\circ} \mathrm{C}$ (fuel temperature panel).

Following Step 4, the corresponding TC1 reading of $961{ }^{\circ} \mathrm{C}$ (TC1 panel) is recorded as the control TC input signal for control TC1 in the capsule temperature control system for time after 264 EFPD.

- For after period (bottom row), the selected parameters are 500 EFPD, the graphite heat rate is $6 \mathrm{~W} / \mathrm{cm}^{3}$, the fuel power density is $80 \mathrm{~W} / \mathrm{cm}^{3}$, and the neon fraction is adjusted to 0.80 for the fuel temperature to reach its desired temperature of $1100^{\circ} \mathrm{C}$. The corresponding $\mathrm{TC} 1$ reading of $929^{\circ} \mathrm{C}$ is recorded as the control TC input signal for control TC1 in the capsule temperature control system for the time period by the end of AGR-1 irradiation.

According to Section 2, Capsule 5 TC1 readings drifted upward in relation to its simulation over the entire AGR-1 experiment for about $200^{\circ} \mathrm{C}$. However, the control TC input signal for the control TC1 at 500 EFPD decreases by only $32^{\circ} \mathrm{C}\left(961\right.$ minus $\left.929^{\circ} \mathrm{C}\right)$ in comparison with $100 \mathrm{EFPD}$. The small decrease in the control TC1 input signal versus the TC1 drift amount at 500 EFPD could be explained by the reduction of the control gas gap on TC readings and fuel temperatures. The big decrease in control gas gap by the end of AGR-1 greatly reduces TC3 readings, while the increase in the gap between the fuel compacts and graphite holder lessen the decrease in fuel temperature. Thus, the bigger reduction of actual TC1 reading is counteracted with TC1 upward drift, leading to small change in the updated TC1 input signal be the end of the AGR-1 test. 

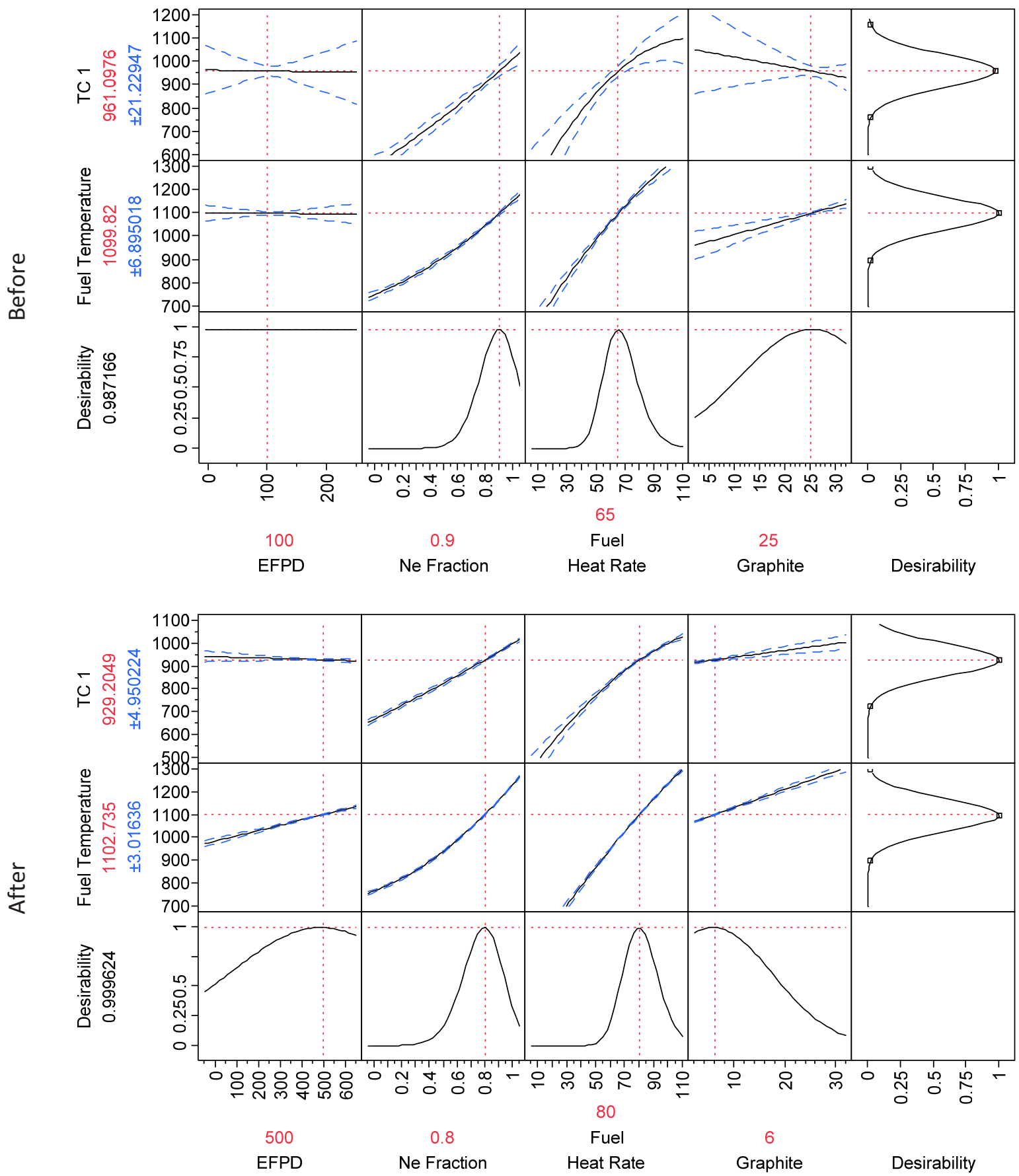

Figure 24. Prediction profilers for TC1 and simulated fuel temperature in Capsule 5. 


\subsubsection{Example 2: Assume TC3 is the Control TC in Capsule 5}

The prediction profilers and desirability functions of fuel power, graphite power, and neon fraction for measured TC3 and simulated fuel temperature are presented in Figure 25. The corresponding control TC3 input signal right after 264 EFPD and by the end of AGR-1 is determined as follows:

- For the before period (top row), the same parameters on 100 EFPD were selected, the graphite heat rate is $25 \mathrm{~W} / \mathrm{cm}^{3}$, the fuel heat rate is $65 \mathrm{~W} / \mathrm{cm}^{3}$, and neon fraction is adjusted to 0.9 to maintain the fuel temperature at $1100^{\circ} \mathrm{C}$ ). The corresponding $\mathrm{TC} 3$ reading is then $875^{\circ} \mathrm{C}$.

- For the after drift period (bottom row): the data on 500 EFPD are also selected, the graphite heat rate is $6 \mathrm{~W} / \mathrm{cm}^{3}$, the fuel power density is $80 \mathrm{~W} / \mathrm{cm}^{3}$, and the neon fraction will be 0.803 . However, the corresponding TC3 reading at this time was dropped to $692^{\circ} \mathrm{C}$.

Since TC3 readings are consistent with its simulation, a big drop of $183^{\circ} \mathrm{C}$ in TC3 input signal at 500 EPFD (from 875 to $692^{\circ} \mathrm{C}$ ) is mostly because of changes in the capsule thermal condition by the end of the AGR-1 test. As stated in Example 1, the big decrease in control gas gap by the end of AGR-1 reduces TC3 readings more than it reduces fuel temperature. Unlike Example 1, TC3 readings are consistent with simulation, so the TC3 input signal should be reduced accordingly to maintain the same fuel temperature.

The above-proposed experimental control procedure, along with the statistical analysis techniques implemented in NDMAS, could be used to improve fuel temperature control in support of new experiments in the AGR series.

Experience from analysis of AGR-1 test data suggests that during the long duration of fuel testing, several to all TCs in individual capsules may fail, leaving the thermal conditions of fuel compacts in such capsules practically unmonitored. Yet, as both the global conditions (operating parameters [lobe power, shim cylinder positions], and the local conditions (fuel/graphite conditions) in the capsule change, the target quantity (e.g. maximum or average fuel temperatures) also vary. It is therefore highly valuable for the test program that the target quantity is still maintained within the desired range. Using the algorithms of regression analysis and correlation analysis, and data from functioning TCs in the same or neighboring capsules, the missing control data (provided by the now-failed TC) can be evaluated. Such a fill-in, although subject to uncertainties, is instrumental in retaining control over the testing conditions in an otherwise unmonitored capsule. 

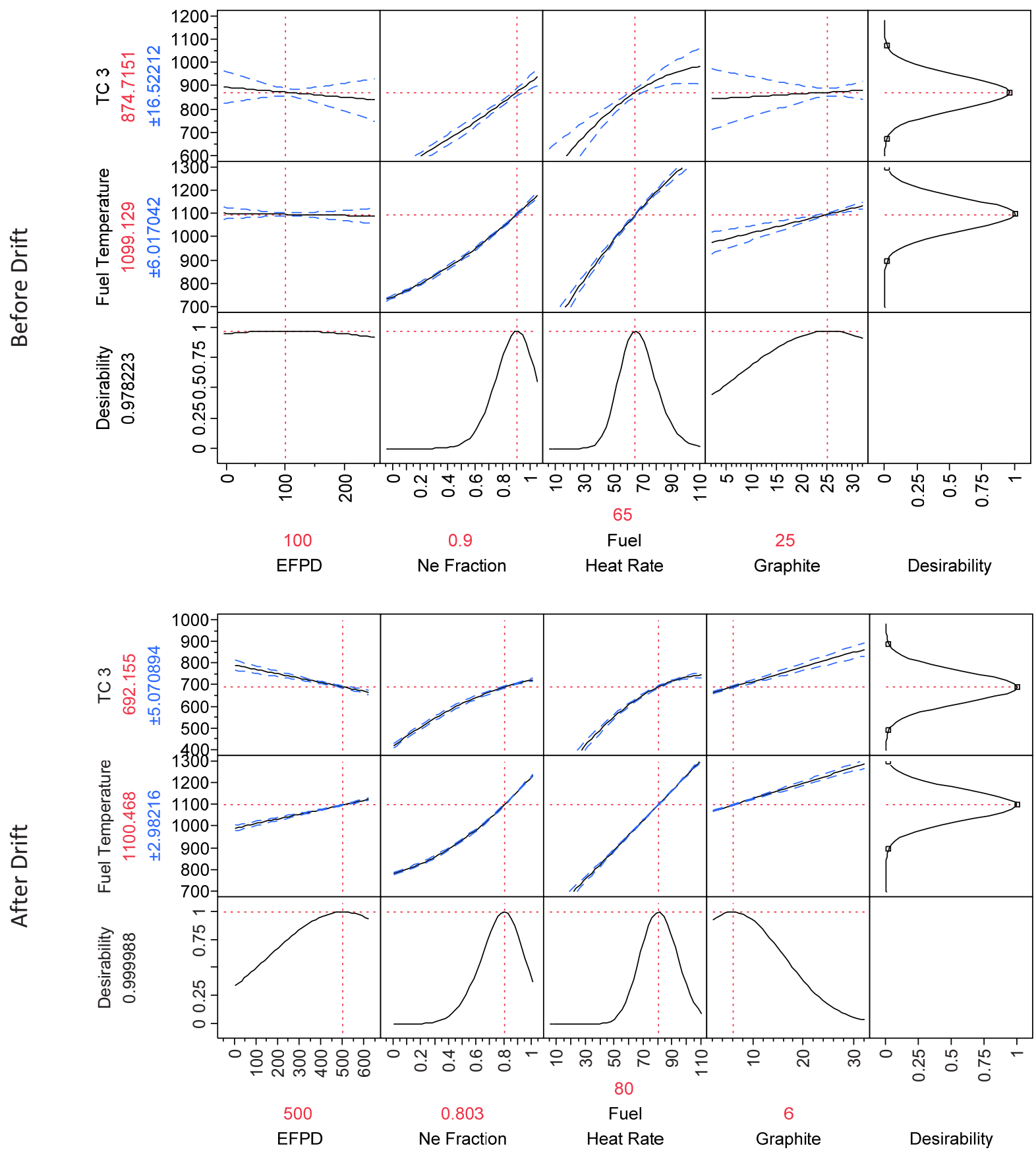

Figure 25. Prediction profilers for TC3 and simulated fuel temperature in Capsule 5. 


\section{CONCLUSION}

Experimental data, particularly data from nuclear fuel performance tests, play an essential role in the design, safety analysis, and licensing of nuclear reactors. Highly valuable data on nuclear fuel performance tests are hard to produce and challenging to interpret. On the one hand, it is critical that the test conditions are effectively maintained during the test and accurately characterized to ensure the experiments meet their objectives. On the other hand, even several decades from now, the experimental data obtained in today's tests may remain valuable for the assessment of models and codes developed to predict nuclear fuel performance that support fuel and plant licensing. As more advanced models and high-fidelity simulation methods become available in the future, their utility is expected to be even more sensitive to knowledge about the quality of data used for model validation and uncertainty quantification. For example, it is important that potentially misleading and deteriorated data from partially failed (drifted, conjunct) TCs be accordingly identified. The techniques and tools developed in NDMAS support this data qualification objective.

Nuclear fuel performance experiments involve complex physical mechanisms such as fissile depletion, graphite and fuel shrinkage, and property (conductivity, density) changes for which the knowledge and capability to accurately assess the impact of modeling assumptions about these processes under AGR conditions are lacking. This greatly complicates the task of separating the contribution from experimentation and simulation to an off-trend observation. The combined use of test data and simulation results therefore requires a systematic approach to uncertainty quantification of both experimental measurements on one side, and model and numerical solutions on the other side. Furthermore, observing trend differences in deemed stable measured TCs and simulated TCs provides insight and guidance in model improvement. The thermal simulation data provides additional evidence, helping to confirm the drifted failure of some TCs in the AGR-1 test. Since the comparative statistical methods could reveal the possible failure of at least one TC in the subject TC pair, the simulation data helped identify the one that is likely to fail when its measurements are not consistent with the simulation. The measurement data also help reveal potential issues in physics and thermal models. For example, the depletion model might underestimate fuel and graphite heat rates toward the end of every ATR cycle and the thermal model might overestimate temperature when neon fraction is increasing, especially when approaching pure neon.

The AGR simulation results collected in the NDMAS can be very useful in aiding experimental data qualification, especially increasing the confidence in delineating failures of the measuring instruments (TCs) from physical mechanisms that may have shifted the system thermal response. Given a high rate of TC failure under harsh irradiation and thermal conditions in AGR, it may be beneficial to utilize the temperature prediction results from the daily AGR power/thermal simulations to provide early detection of changes (drifting) or failure of TCs and support a more effective test control procedure. Thus, it is appealing that the statistical methods developed for TC drift detection discussed in Chapter 2 can not only can be used for post-test data qualification but also to increase the test quality via regulation of control parameters in the active experiment. 


\section{REFERENCES}

Abbott, M. L., et al., 2010, AGR-1 Data Qualification Report, Idaho National Laboratory, INL/EXT-10-17943.

BNL, 2009, Upton, NY: BNL-XXXXX-2009.

Hawkes, G. L, 2012, “AGR-1Daily As-run Thermal Analyses,” Idaho National Laboratory, ECAR-968, Rev. 2.

Hawkes, G. L., Sterbentz, J.W, \& Maki, J.T., 2011, "Sensitivity Evaluation of the Daily Thermal Predictions of the AGR-1 Experiment in the Advanced Test Reactor," paper \# 11186, International Congress on Advances in Nuclear Power Plants (ICAPP 2011), May 2-5, 2011 - Nice, France

INL, 2009a, "Very High Temperature Gas Reactor Technology Development Office Quality Assurance Program Plan," Idaho National Laboratory, PLN-2690, Rev 3.

INL, 2009b, "Very High Temperature Reactor Program Data Management and Analysis Plan," Idaho National Laboratory, PLN-2709.

INL, 2009c, "Temperature Control and Off Gas Monitoring Systems for Advanced Gas Reactor Experiment AGR-1," Idaho National Laboratory, TFR-248, Rev. 3.

INL, 2010, "VHTR Technology Development Office Program Management Plan,” Idaho National Laboratory, PLN-2494.

Maki, J., 2004. AGR-1 Irradiation Test Specification, Idaho National Laboratory, EDF-4731, Rev. 11.

Maki, J., 2009, AGR-1 Irradiation Experiment Test Plan, Idaho National Laboratory, INL/EXT-0500593, Rev. 3.

Petti, D., Hobbins, R., \& Kendall, J., 2010, Technical Program Plan for the Next Generation Nuclear Plant/Advanced Gas Reactor Fuel Development and Qualification Program. Idaho National Laboratory: INL/EXT-05-00465, Rev. 3.

Pham, B.T., and Einerson, J.J., 2010, "Statistical Analysis Support for Nuclear Fuel Performance Experimental Data Qualification," ANS Conference Proceeding, June 13-17, 2010, San Diego, CA.

SAS, 2007, "SAS Enterprise Business Intelligence Server,” Cary, NC, SAS Institute Inc.

SAS, 2009, JMP 8 Statistics and Graphics Guide, Second Edition, Cary, NC, SAS Institute Inc.

Simonds, J., 2010, “Technical Program Plan for the Next Generation Nuclear Plant/Advanced Gas Reactor Fuel Development and Qualification Program," Idaho National Laboratory, PLN-3636 R.0.

Sterbentz, J. W., 2011, "JMOCUP As-Run Daily Depletion Calculations for the AGR-1 Test in the ATR B-10 Position,” Idaho National Laboratory, ECAR-958, Rev. 1. 